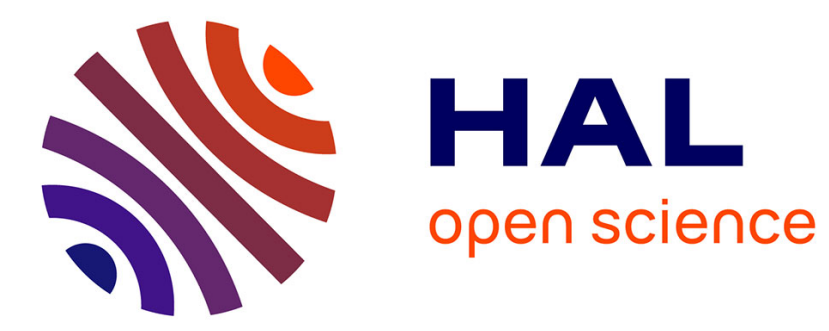

\title{
Aeroacoustic source analysis in a corrugated flow pipe using low-frequency mitigation
}

\author{
Muriel Amielh, Fabien Anselmet, Yuchao Jiang, Ulf R. Kristiansen, \\ Pierre-Olivier Mattei, Daniel Mazzoni, Cédric Pinhede
}

\section{To cite this version:}

Muriel Amielh, Fabien Anselmet, Yuchao Jiang, Ulf R. Kristiansen, Pierre-Olivier Mattei, et al.. Aeroacoustic source analysis in a corrugated flow pipe using low-frequency mitigation. Journal of Turbulence, 2014, 15 (10), pp.650-676. 10.1080/14685248.2014.928412 . hal-01057978

\section{HAL Id: hal-01057978 https://hal.science/hal-01057978}

Submitted on 14 Jan 2022

HAL is a multi-disciplinary open access archive for the deposit and dissemination of scientific research documents, whether they are published or not. The documents may come from teaching and research institutions in France or abroad, or from public or private research centers.
L'archive ouverte pluridisciplinaire HAL, est destinée au dépôt et à la diffusion de documents scientifiques de niveau recherche, publiés ou non, émanant des établissements d'enseignement et de recherche français ou étrangers, des laboratoires publics ou privés.

\section{(ㄷ)(1) $\$$}

Distributed under a Creative Commons Attribution - NonCommerciall 4.0 International 


\title{
Aeroacoustic source analysis in a corrugated flow pipe using low-frequency mitigation
}

\author{
M. Amielh 1, F. Anselmet ${ }^{1,2}$, Y. Jiang 3 , U. Kristiansen 3 , \\ P.O. Mattei ${ }^{4}$, D. Mazzoni 4,2, C. Pinhède 4 \\ 1 - Aix Marseille Université, CNRS, Centrale Marseille, IRPHE UMR 7342, 49 rue F. Joliot-Curie, \\ 13384 Marseille Cedex13, France \\ 2 -ECM, 38 rue F. Joliot-Curie, 13451 Marseille Cedex 13, France \\ 3 - NTNU, Acoustic Research Centre, O. S. Bragstads plass 2A, 7034 Trondheim, Norway \\ 4 - LMA, CNRS, UPR 7051, 31 chemin Joseph Aiguier, 13402 Marseille Cedex 20, France \\ Corresponding author : anselmet@irphe.univ-mrs.fr
}




\title{
Aeroacoustic source analysis in a corrugated flow pipe using low-frequency mitigation
}

\begin{abstract}
Our study is focused on a phenomenon often encountered in flow carrying pipes, since flow instabilities caused by geometric features may generate acoustic signals and thereafter interact with these signals in such a way that powerful pure tones are produced. A modern example is found in the so-called "singing risers", or the gas pipes connecting gas production platforms to the transport network. But the flow generated resonance in a fully corrugated circular pipe may be silenced by the addition of relatively low frequency flow oscillations induced by an acoustic generator. Experiments reported here, aimed at investigating in more detail the coupling between the flow in the pipe, the acoustically generated flow oscillations and the emitted resulting noise, are performed in a specifically designed facility. A rectangular transparent channel using glass walls enables us to use optical techniques to describe in detail the flow field in the corrugation vicinity, in addition to more standard hot-wire anemometry and acoustic pressure measurements with microphones, with and without the acoustically generated low-frequency oscillations.
\end{abstract}

Keywords : Corrugated pipe; acoustic mitigation; turbulent flow 


\section{Introduction}

Due to their flexibility and local stiffness, corrugated pipes are used in many engineering applications. It is known that if the pipes carry a flow of dry gas, whistling sounds associated with the pipes' length resonances may develop. This phenomenon is used positively in musical toys like the Hummer [1,2], but represents a problem in engineering applications as high sound levels and associated structural vibrations may develop. A well-known example is the "singing riser" phenomenon observed on some natural gas installations in the North Sea.

Scientific study of the phenomenon dates back almost 100 years. Burstyn [3] discussed briefly the phenomenon at the 1921 meeting of the German Society for Technical Physics. Around the same time, Cermak [4] studied the tones produced in short $(0.5 \mathrm{~m})$ tubes having a narrow bore. He established that the frequencies excited are a little lower than the ones expected in smooth pipes, and that this could not be an effect of the end conditions. He also found that the frequencies heard correspond approximately to the "bump" frequencies based on flow velocity and corrugation pitch. More recent investigations show that the frequency effect is due to a lowering in the wave speed caused by the acoustic stiffness of the cavities ([5], see also section 3.2), and that the phenomenon occurs at a constant Strouhal number if the length parameter is taken as the cavity width plus the radius of curvature of the upstream cavity edge [6]. Examples of experimental studies giving information on Strouhal and Reynolds numbers, necessary flow velocities, discussions on source positions, etc, for different test pipes, are found in references [7 - 11]. The works cited above are performed on relatively short narrow bore pipes. A study directly related to the singing riser problem is the one by Belfroid et al. [12].

Theoretical work on the subject includes the work by Debut et al. [13] who modelled the acoustic sources as a distribution of van der Pol oscillators along the pipe, and Goyder [14] who studied the distribution of sources and sinks along an acoustic wavelength in a corrugated pipe. More direct numerical studies have also appeared in recent years. Popescu et al. [15] used a turbulent model in Fluent to study the aero acoustic interaction in a short pipe. In parts of the pipe they found a strong interaction between cavity vortices and the shear layer separating the cavity fluid from the free stream. Nakibouglou et al. [2,6] modelled the flow in a pipe section including cavities using an incompressible Fluent version with a superposed oscillation corresponding to the frequency of a pipe mode. Their studies quantified the amount of sound power generated by the interaction of vortices shed at the cavities leading edges and the acoustic field.

Related to this, a study by Kopiev et al. [16] should be noted. In their study, a broad band signal is generated by a loudspeaker in a plenum chamber at one end of a corrugated pipe. They demonstrated that the acoustic signal would increase or decrease when propagating through the pipe depending on frequency and flow velocity.

Flow above single cavities with an associated sound production is a well-established field of study [17]. Of special interest to the present study is the finding that in cases where the pipe's entry section is sharp enough for the flow to separate, a small cavity a short distance downstream may give rise to strong longitudinal pipe modes [18, 19]. The distance between the pipe's entry section and the single cavity's downstream corner is thought to be the relevant distance for Strouhal number estimations. As the corrugated test pipe used in the present study also has a sharp edged, partly flanged, entry section, it is expected that this will represent a possible source region.

The interested reader will also benefit from the recent review by Rajavel and Prasad [20]. This paper summarizes the main available theories used to describe the singing phenomenon in corrugated pipes. It also reviews various experimental and computational works carried out 
for this problem and highlights remaining open questions such as the missing of the fundamental tone of corrugated pipes and the lack of prediction tools for the whistling effective acoustic sound pressure level.

The objective of the present research is to study both the acoustic and aerodynamic fields within a corrugated plane channel under singing conditions using both synchronised acoustic pressure and velocity measurements and particle image velocimetry (PIV).

We want to investigate more fully a previously published finding, mainly that adding a strong signal at a frequency very much lower than the whistling frequency of the channel, under some conditions, will quench the whistling [21]. In this previous study, an intermittent residual whistling noise remained. This whistling appeared and disappeared within a cycle of the low frequency signal. In the present study, we want to use this residual whistling in order to analyse the aeroacoustic source.

A model of the flow in a corrugated plane channel, based on a lattice Boltzmann technique, suitable for compressible flow modelling at low Mach numbers is faced to experimental results.

The paper is organized in the following way: section 2 describes the experimental arrangement and procedures, section 3 is devoted to the identification of sound pressure field characteristics inside the channel, section 4 reports the corrugated channel flow main characteristics, section 5 presents the analysis of the experimental results, with particular attention to the acoustic mitigation, section 6 presents the Lattice Boltzmann simulations, while section 7 presents discussion of the Lattice Boltzmann results. Finally, the final discussion and conclusion are presented in section 8 .

\section{Experimental arrangement}

The experimental facility under study is made of a low speed wind tunnel whose fan rotation speed ensures a flow velocity up to $25 \mathrm{~m} / \mathrm{s}$. The tunnel is connected to a corrugated rectangular channel of length $L=2 \mathrm{~m}$, prolonged by a loudspeaker itself ended by an open resonator of $17 \mathrm{~m}$ length in order to obtain high level acoustic oscillations at low frequency.

The rectangular corrugated channel is made of Plexiglas plates allowing direct inspection of the aerodynamic field by a PIV technique. The corrugated channel has lateral width $D=20 \mathrm{~mm}$ and height $B=100 \mathrm{~mm}$. The lateral plates are machined in order to make a wall with a rectangular corrugation of pitch length $P t=20 \mathrm{~mm}$ that is repeated all along the channel. The corrugation occupies the full height of the lateral walls of the channel. As shown in Figure 1, the corrugations are rectangular with a depth $H=10 \mathrm{~mm}$, and a width $w=10 \mathrm{~mm}$; the upstream corner of the corrugation is rounded with a curve with curvature radius $r=2.5 \mathrm{~mm}$. The top and bottom walls of the channel are perfectly smooth. The corrugations occupy about $83 \%$ of the wall surface of the channel. 

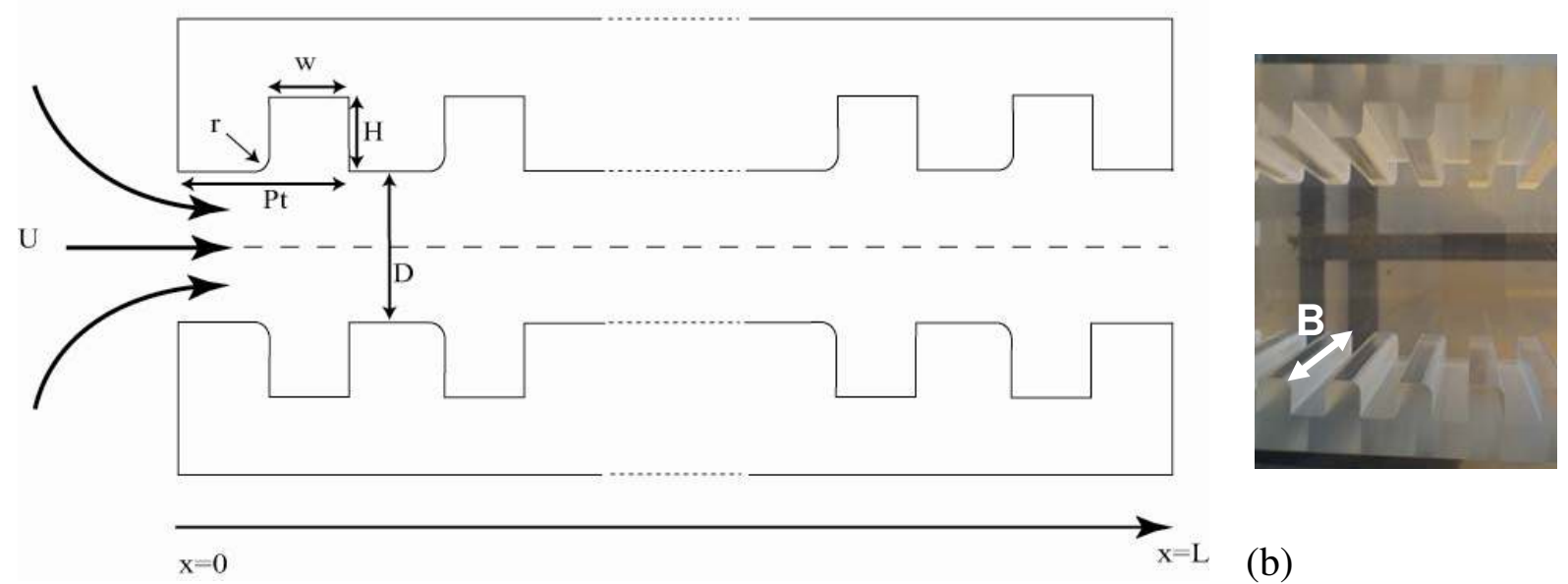

(b)

(a)

Figure 1. Geometry of the corrugated channel. a) Schematic view, b) Photography of details of the face to face vertical corrugated plates.

The entry of the corrugated channel is connected to a fan, as indicated in Figure 2, using a rectangular coupling box (see tick (1) in Figure 2). This box ensures, not only air tightness of the system corrugated channel/fan, but also that the channel has acoustically open flanged ends. The exit end of the corrugated channel is connected to a loudspeaker coupling box (see ticks(5) and (6) in Figure 2) extended by the $17 \mathrm{~m}$ resonator whose resonance frequency is around $10 \mathrm{~Hz}$. This resonator ensures that there is a strong coupling between the corrugated channel and the loudspeaker around its resonance frequency.

The loudspeaker is made of two push-pull sub-woofers SONIC-10MK2 (bandwidth $f \in[3,300] \mathrm{Hz}$ ), mounted face to face and supplied with the same in-phase signals from a power amplifier ECLER MPA 4-150R.

Velocity measurements are performed by hot-wire anemometry for temporal/spectral analysis and by PIV for spatial analysis. Two single hot-wires (DANTEC 55P11, $1.2 \mathrm{~mm}$ length, $5 \mu \mathrm{m}$ diameter) are used to characterise the flow velocity: the first one is located $10 \mathrm{~mm}$ upstream of the vein entry (see tick (1) in Figure 2 and Figure 3) and the other one is downstream, inside the vein, $19 \mathrm{~mm}$ just upstream of its exit section (see tick (2) in Figure 2). Hot-wire anemometry is controlled by the DANTEC Streamline system using the Streamware software. When not synchronously acquired with microphone signal by the Metravib NetdB system (24 bits), the hot-wire analogous signals are acquired by a 12 bit acquisition data card with a frequency of $25 \mathrm{kHz}$. For each hot-wire, $2^{20}$ samples are acquired. 


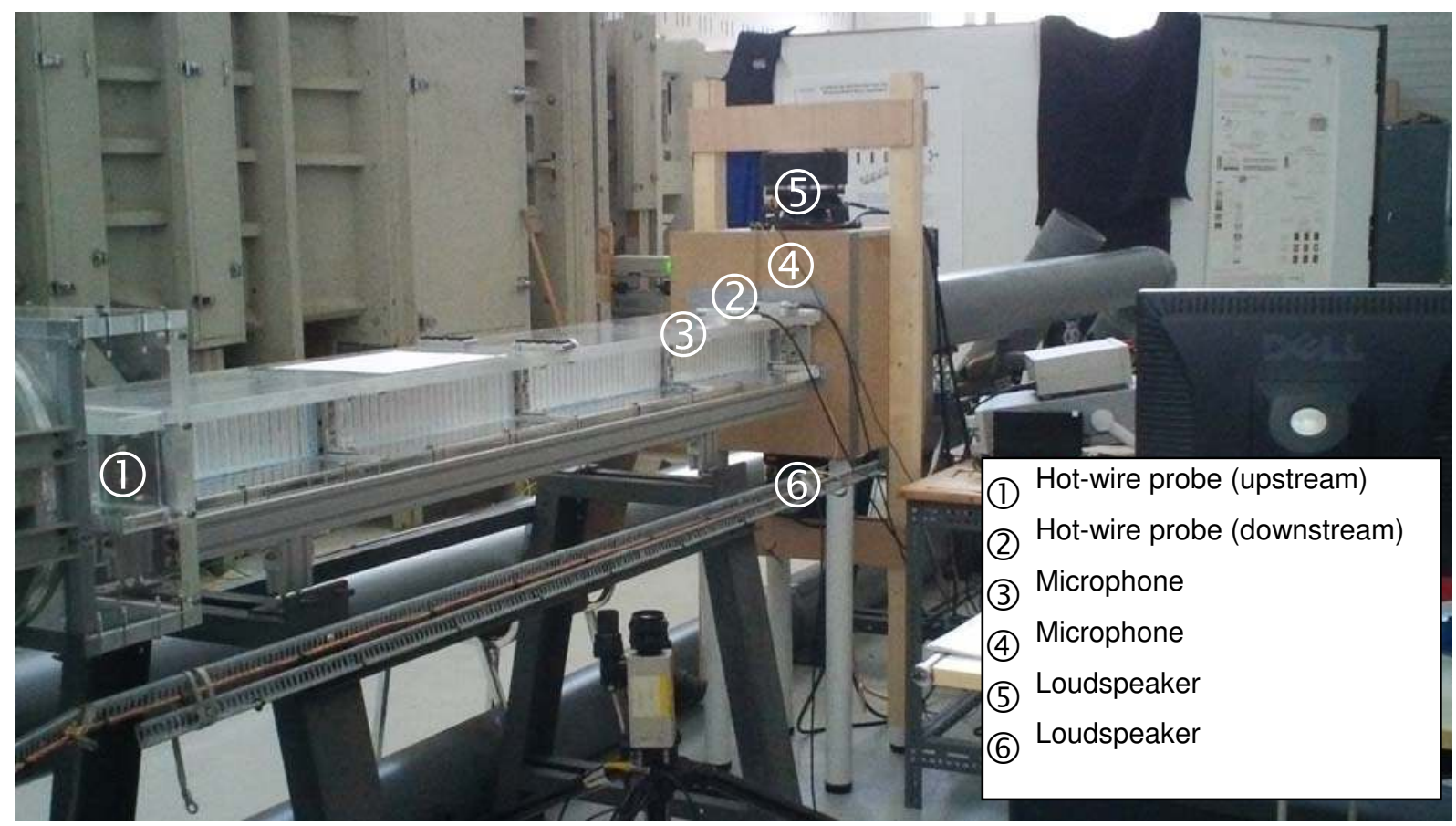

Figure 2. Photography of the experimental arrangement. Exit part of the corrugated channel and loudspeaker mitigation system; the fan is located on the left of the photography.

Hot-wire measurements are used to obtain the relation between the fan motor rotation and the flow velocity inside the vein (Figure 4). The upstream hot-wire is located in the vena contracta that feeds the corrugated channel, so that the velocity detected by this probe is lower than that measured by the downstream hot-wire.

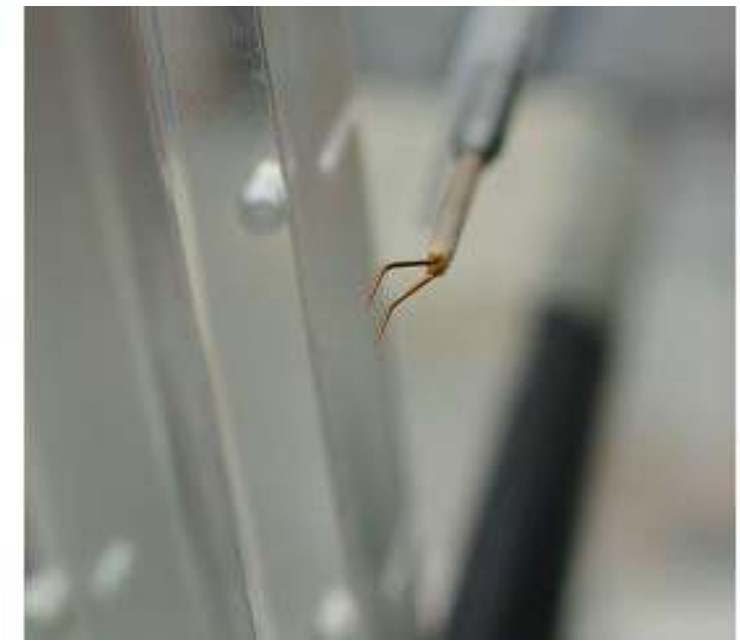

Figure 3. Photography of the entry hot-wire.

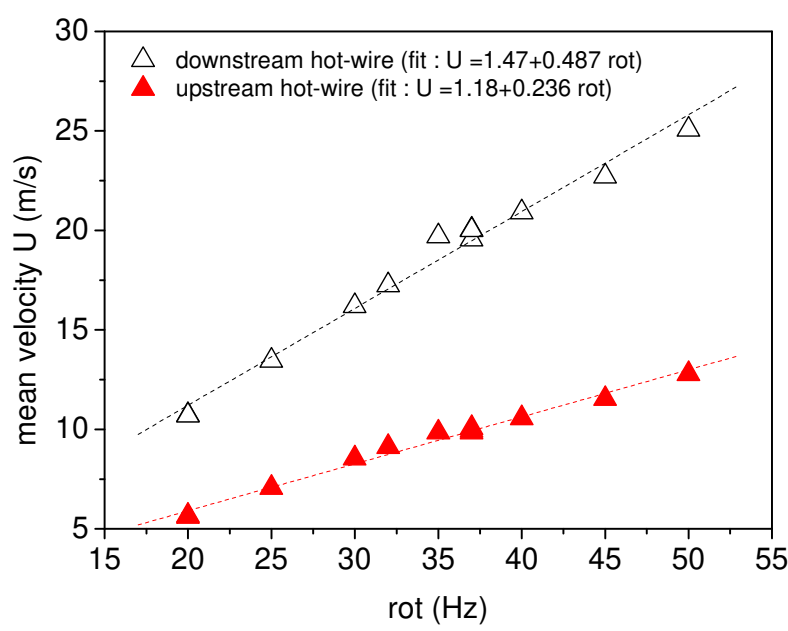

Figure 4. Relation between the fan motor rotation and the mean velocity at the entry and exit of the corrugated channel measured by hot-wires.

PIV is used to characterize the velocity field inside a cavity. The investigated cavity is the $35^{\text {th }}$ one, located at $700 \mathrm{~mm}$ from the vein entry, ie at approximately one third of the vein length. Since the acoustic sound pressure inside the channel shows a succession of maxima and minima, (see Figure 6), this particular location was chosen because it is in the vicinity of a minimum of the sound pressure corresponding to a maximum of the acoustic velocity for the 
whistling frequencies of interest. According to the axial pressure measurements presented hereafter, this cavity is located near a minimum of pressure level when the fan motor rotation is $r o t=37 \mathrm{~Hz}$. The PIV system includes a pulsed Yag laser (Spectra-Physics, 200mJ, $10 \mathrm{~Hz}$ ) and a KODAK camera with $1008 \times 1018$ pixels. The whole system is synchronized by a Stanford synchronizer. The field of view is about $12 \mathrm{~mm} \times 12 \mathrm{~mm}$, centred on the cavity. For each experiment, 1500 pairs of images are acquired with a $10 \mathrm{~Hz}$ rate and with a time delay for a pair of images in the range $12-15 \mu \mathrm{s} .1500$ velocity fields are processed with the Dynamic Studio software (DANTEC Dynamics). The size of interrogation windows is $32 \times 32$ pixels, with an overlap value between 50 and $75 \%$ depending on the required accuracy in flow analysis.

Acoustic pressure is measured by microphones (see ticks (3) and (4) in Figure 2). A microphone probe (tick (3) GRAS 40-SC is located at the centre of the corrugated channel inside it, at various positions starting $4.9 \mathrm{~cm}$ upstream of the exit end, up to $28.9 \mathrm{~cm}$ upstream of the exit end, each one separated by $1 \mathrm{~cm}$. The second microphone $(1 / 4$ inch GRAS 40BP, tick (4)) is fixed inside the box, at $10 \mathrm{~cm}$ from the top of the box, for continuous monitoring of the loudspeaker induced pressure level. As shown below, these locations ensure that both probes are able to measure the maximum fluctuations of acoustic velocity and pressure. All pressure level acquisitions have been made by a 01-dB Metravib NetdB acquisition front-end unit using $12.8 \mathrm{kHz}$ sampling rate and 24 bit resolution. For special experiments, both hot-wire signals are simultaneously acquired with similar conditions. All signals have been normalized so that measurements are given in physical units ( $\mathrm{Pa}$ for acoustic sound pressure and $\mathrm{m} / \mathrm{s}$ for velocity).

\section{Characterisation of the sound pressure field inside the pipe}

\subsection{Modal identification}

Both ends of the corrugated pipe can be considered as open. Acoustic boundary conditions can then be described as Dirichlet conditions including a classical correction length to account for sound radiation [21,22]. Such pipes present a discrete set of modes. Each mode is associated to a frequency $f_{n}$ given by $f_{n}=n \frac{c_{\text {eff }}}{2(L+2 \delta L)}, n=1,2, \ldots . c_{\text {eff }}$ is the effective sound celerity in the corrugated pipe, it is slightly lower than the sound celerity inside the fluid due to the corrugations [26], and $\delta L$ is the correction length.

To our knowledge, no theory gives a sufficiently precise value of $c_{\text {eff }}$ for the present configuration. An estimate is proposed here through experimental investigation.

The first step consists in measuring the acoustic pressure at the 25 different positions described above. This is achieved by maintaining the fan rotation of $37 \mathrm{~Hz}$ that gives a constant flow speed of about $19.5 \mathrm{~m} / \mathrm{s}$ inside the corrugated channel, allowing it to "whistle" at $592 \mathrm{~Hz}$. Each record has a duration of $60 \mathrm{~s}$. The $25 L_{p_{j}}$ rms pressure levels are estimated using the usual formula (1)

$$
L_{p_{j}}=10 \log \frac{1}{N} \sum_{i=1}^{i=N}\left(p_{j}\left(t_{i}\right)\right)^{2},
$$

where $p_{j}\left(t_{i}\right)$ is the acoustic pressure at instant $t_{i}$, at point $x_{j}\left(x_{0}=195.1 \mathrm{~cm}, x_{1}=194.1 \mathrm{~cm}, \ldots\right.$, $\left.x_{24}=171.1 \mathrm{~cm}\right)$, where the origin of abscissa $x=0$ is the entry end of the channel. $N=2^{18}$ ensures a nearly perfect estimate of the rms pressure level (the difference between the computation made with $N=2^{18}$ and with $N=2^{19}$ is about $0.05 \mathrm{~dB}$ ). 
An example of the spectrum of the recorded signal at $x=175.1 \mathrm{~cm}$ is given in Figure 5, showing that the main dominant pressure peak has a frequency of about $592 \mathrm{~Hz}$ with a sound pressure level $L_{p}=23.7 \mathrm{~dB}(\mathrm{ref} 1 \mathrm{~Pa})$ while the sound pressure level of the whole signal is close to $L_{p_{20}}=27.4 \mathrm{~dB}$ (ref $1 \mathrm{~Pa}$ ). Even if this location is close to the sound pressure minimum, this measurement shows that the signal to noise ratio is sufficient to ensure a good estimate of the whistling. The correction length $\delta L$ has been estimated by fitting the experimental results with the equation (2):

$$
L_{p p}=10 \log P_{M}^{2} \sin \left(2 n \pi \frac{x}{2(L+2 \delta L)}\right)^{2},
$$

where $P_{M}^{2}$ is the amplitude measured, $n=8$ indicates that the mode under consideration is the 8-th channel longitudinal mode. One obtains $\delta L=1 \mathrm{~cm}$ as the correction length of the channel.

A comparison is presented in Figure 6 between the measured pressure and the identified one.

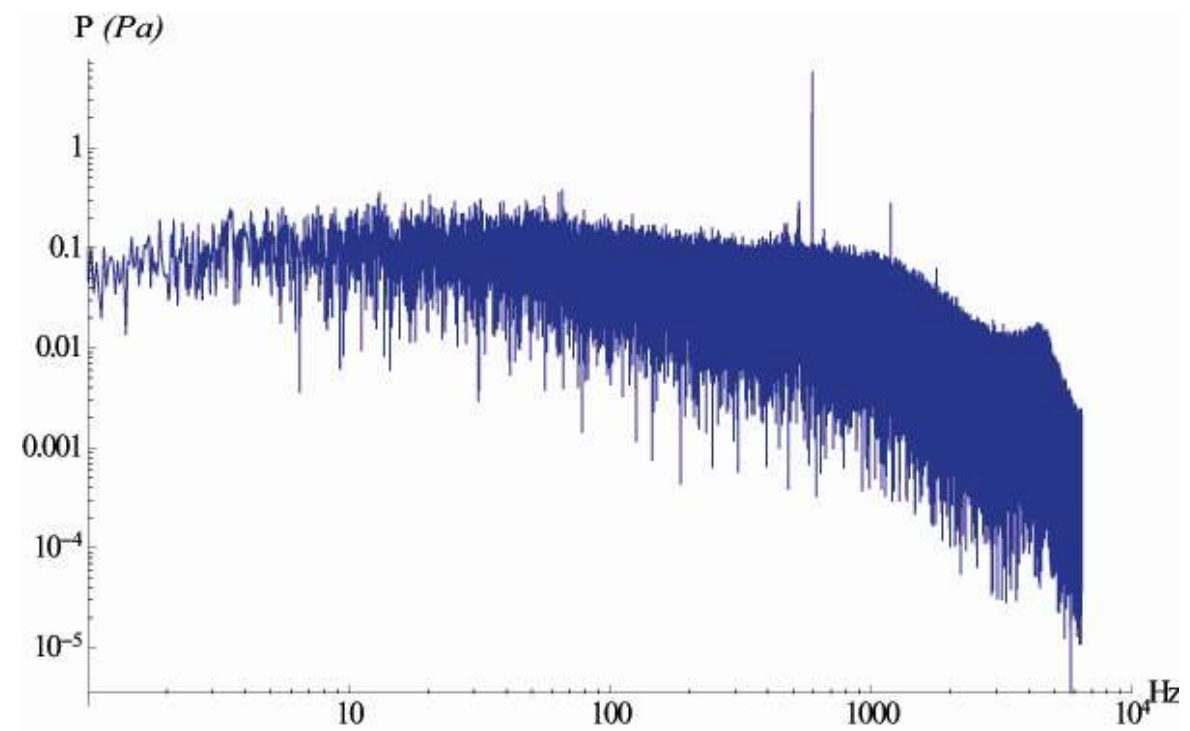

Figure 5. Spectrum of acoustic sound pressure at position $x=175.1 \mathrm{~cm}$ for a fan rotation of $37 \mathrm{~Hz}$.

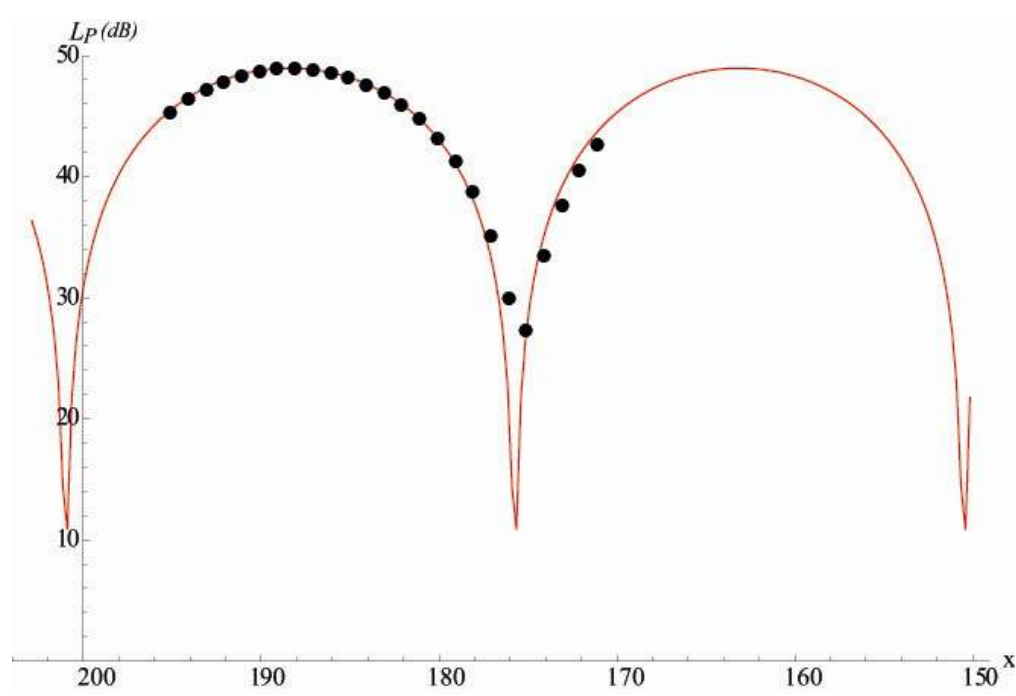

Figure 6. Variation of the pressure along the corrugated channel $(\mathrm{cm})$ for a fan rotation of 37 $\mathrm{Hz}$, Black dots: measurement, red curve: identification. 
This correction length is the simplest way to account for the effect of sound radiation. At low frequency for flanged circular pipe of radius $R$, the correction length is given by the well known result obtained by Rayleigh [23] :

$$
\delta L=0.82 R
$$

In the rectangular case, such a simple result is not known [24, 25]. Nevertheless, it is possible to obtain accurate estimate of correction length for a rectangular channel by using results reported by $[24,25]$. For the presently considered rectangular geometry, with an aspect ratio $\mathrm{B} / \mathrm{D}=5$, from the numerical results presented in Figure 6 of [25], one obtains:

$$
\frac{\delta L}{B / 2} \approx 0.35
$$

from which the correction length is $\delta L \approx 17.5 \mathrm{~mm}$, which is in reasonably good agreement with the value estimated from relation (2) if one considers that the present channel is connected to the loudspeaker coupling box and not to an infinite open space as assumed in [25]. This validates the experimental identification of the mode 8 . From this, one can deduce the experimental effective sound celerity.

\subsection{Effective sound celerity}

It is well known that for acoustically rigid wall corrugated channels, the wave propagates along the channel at a slower, compared to a smooth one (i.e. non corrugated channel), apparent celerity $c_{\text {eff }}[25,26]$. This effective sound speed can easily be deduced from experiments. The measurements show that the dominant frequency is close to $592 \mathrm{~Hz}$. Since it corresponds to the eighth mode, the measured effective sound speed $c_{e f f}^{m}$ is computed by

$$
c_{e f f}^{m}=592 \frac{2(L+2 \delta L)}{8} \approx 299 \mathrm{~m} / \mathrm{s} .
$$

Since the measurements are made at $T=293{ }^{\circ} \mathrm{K}$, the true sound wave celerity is given by $c_{0} \approx 20 T^{1 / 2}=342 \mathrm{~m} / \mathrm{s}$. This apparent contradiction is based on the fact that inside the channel, it is difficult to define a clear propagating path for the wave since the apparent length of a corrugated channel is much longer than the corresponding smooth one. The simplest way to account for this is to use the model proposed by [26]. It considers that the corrugations act as if the walls of the channel were covered with spring-like, purely imaginary impedance with no energy loss/gain. This gives a value of $c_{\text {eff }}$ given, when the Mach number influence is neglected, by [26, p. 219, eq. 50-53]

$$
c_{\text {eff }}=c_{0} \frac{1}{\sqrt{1+\frac{P}{S} \frac{w}{P t} \frac{V_{c}}{S_{p}}}},
$$

where $P=2(B+D)$ is the perimeter of the tube, $S=D . B$ the section of the tube, $V_{c}=2 w . H . B$ is the volume cavity, $S_{p}=2 w . B$ is the area presented by the cavity to the channel and $P t$ is the pitch length. With the experimental geometric data, one obtains an apparent velocity $c_{e f f} \approx 270 \mathrm{~m} / \mathrm{s}$ that is clearly underestimated compared to the measured one, $c_{\text {eff }}^{m}$ given by equation (5). This approximation can be improved by considering that this model supposes that the corrugations occupy the whole section of the channel, while in our experiments the corrugations occupy only $83 \%$ of the inner surface of the channel since its top and bottom parts are smooth. A second improvement is obtained by noting that the spring-like effect of the cavity depends strongly on its size and, as the upstream edge of the cavity is rounded with a curvature radius $r$, then, the area presented by the cavity to the channel must be corrected to $S_{p}=2(w+r) . B$. Then, one obtains 


$$
c_{\text {eff }}=c_{0} \frac{1}{\sqrt{1+0.83 \frac{w \cdot H}{P t} \frac{w}{w+r} \frac{2(B+D)}{D . B}}},
$$

which gives an effective sound celerity $c_{\text {eff }} \approx 288 \mathrm{~m} / \mathrm{s}$ close to that deduced from experiments, $c_{e f f}^{m}$.

This formula can be used to estimate the effective sound celerity in rectangular partially corrugated channels.

\section{Flow description}

Since the whistling of corrugated pipes is induced by flow inside the pipe, let us describe its characteristics.

The flow velocities measured at the vein exit are varied in the range $0-25 \mathrm{~m} / \mathrm{s}$ according to the rotation frequency of the fan motor (Figure 4). For the previously described arrangement, the whistling begins to be detected on the upstream hot-wire signal at $16 \mathrm{~m} / \mathrm{s}$, corresponding to a fan rotation of around $r o t=30 \mathrm{~Hz}$. The upstream hot-wire captures the pure tone of the oscillations while the turbulence that develops all along the corrugated channel is superimposed to this sinusoidal oscillation on the downstream hot-wire signal (Figure 7). The whistling is associated to both a strong amplification of the velocity root-mean square measured by the upstream hot-wire and an increase of the sound pressure level from 50 up to $110 \mathrm{~dB}$ (Figure 8).
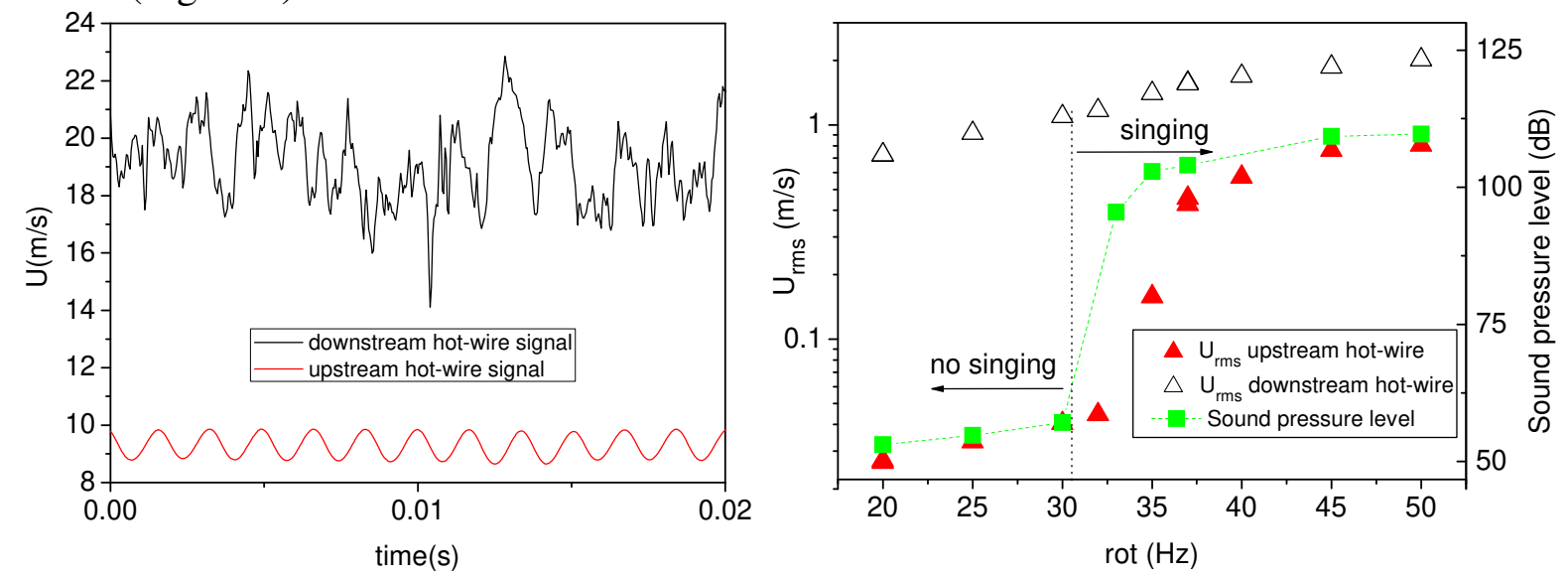

Figure 7. Response of the corrugated channel to an internal flow. Velocity time series from hot-wires with whistling.

Figure 8. Response of the corrugated channel to an internal flow. Amplification of $\mathrm{U}_{\mathrm{rms}}$ measured by hot wire at the vein entry and sound pressure level measured by microphone inside the vein.

The response of the system in presence of a flow is checked to be in agreement with the predicted eigen-frequencies. The whistling is detected on both velocity and pressure signals for which spectral analyses associate the strong values observed in figure 8 to the frequencies associated to $6^{\text {th }}$ to $9^{\text {th }}$ modes as shown in Figure 9 . In this Figure, the Strouhal number based on these main peak frequencies $f$, the characteristic dimension $(w+r)$ and the mean velocity $U$ measured by the downstream hot-wire is shown to be constant around a mean value of $S t=0.36$. This value is in agreement with the peak-whistling Strouhal numbers registered in corrugated pipes in the range 0.32-0.5 [27]. As indicated by Lattice Boltzmann simulations of the flow over a single cavity [19], the noise emission occurring when a corrugated pipe is submitted to an internal flow is associated to the formation of vortices. These vortex 
structures are convected by the flow to the downstream edge of the cavity and contribute to give a non-null time averaged acoustic power.

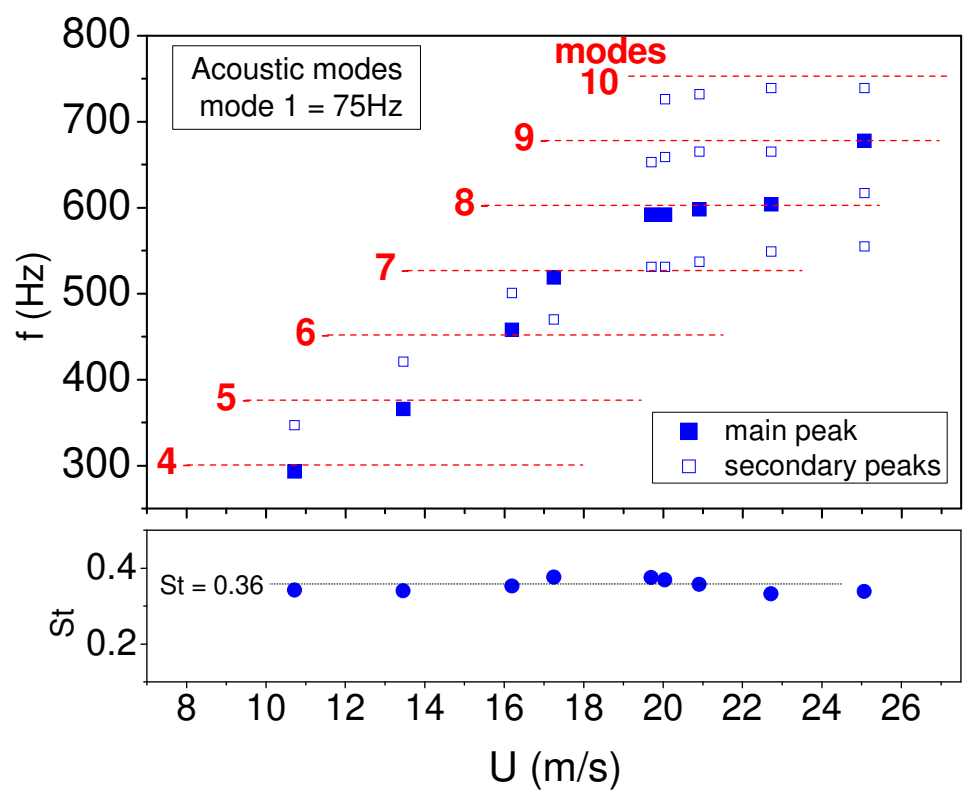

Figure 9. Response of the corrugated channel to an internal flow. Whistling frequencies (from hot-wire measurements) and associated Strouhal numbers, $S t=f(w+r) / \mathrm{U}$.

PIV measurements are used to investigate the flow inside a cavity and detect possible structures. The instantaneous PIV fields show the presence of a vortex that occupies the whole cavity, but no advected structures are highlighted, probably due to the low data acquisition rate of PIV. The statistical analysis indicates that the mean vortex is almost centred in the cavity (Figure 10).

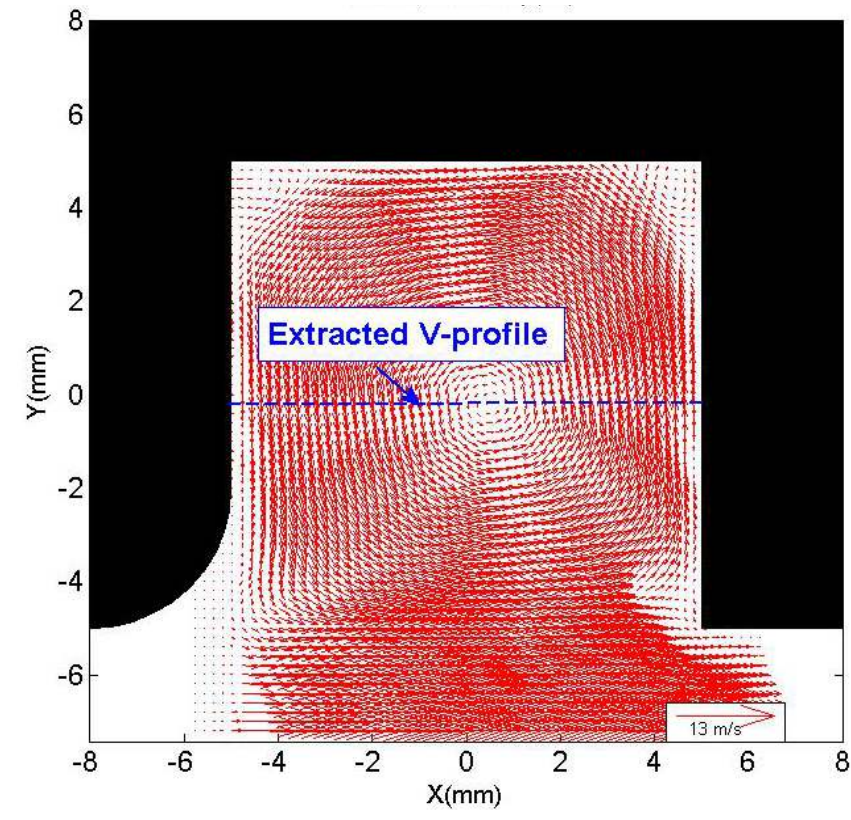

Figure 10. Mean vortex in the cavity: Velocity vectors measured by PIV and location of the extracted V-profiles.

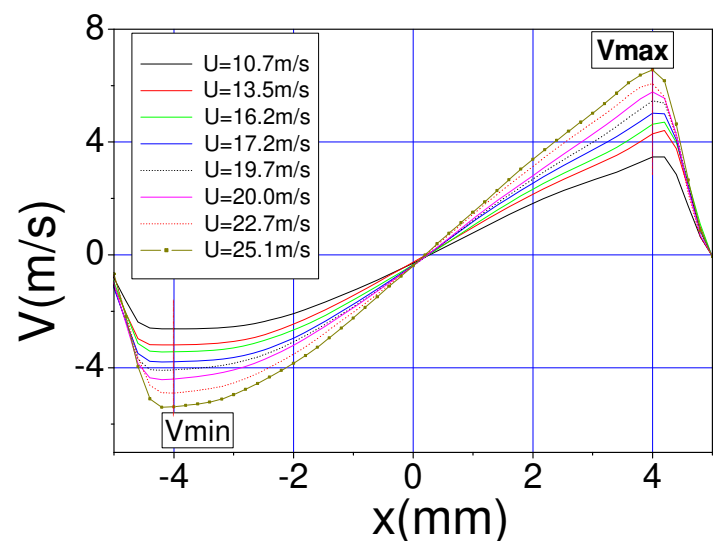

Figure 11. Mean vortex in the cavity: extracted mean velocity profiles from PIV measurements. 


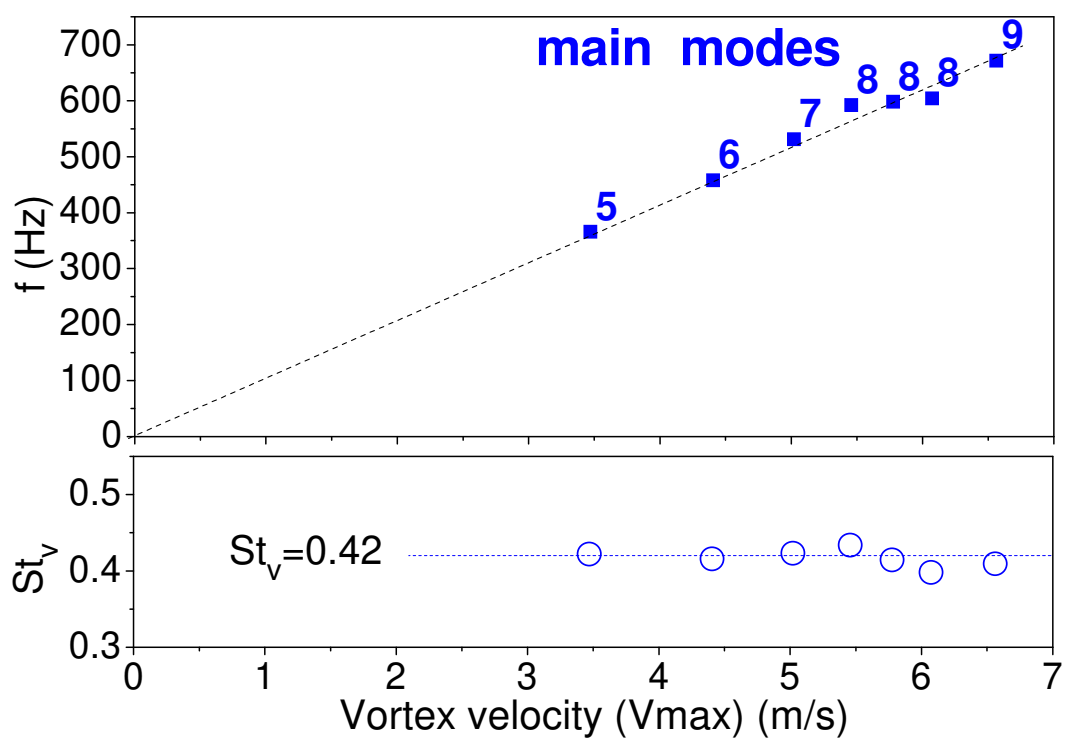

Figure 12. Mean vortex in the cavity: associated modes detected by hot-wire measurements and Strouhal number based on the vortex characteristics: $S t_{v}=f R_{\text {vortex }} / V_{\max }$.

The profiles of the mean vertical velocity of the vortex inside the cavity are extracted for each flow condition and are presented in Figure 11 . The maximal velocity $V_{\max }$ detected on each profile can be related to the modal frequency by the building of a new Strouhal number, $S t_{v}=f R_{\text {vortex }} / V_{\max }$. This Strouhal number is almost constant around $S t_{v}=0.42$ (Figure 12) when $R_{\text {vortex }}=4 \mathrm{~mm}$ is the mean radius of the vortex deduced from the location of the extrema of $V$ in Figure 11. Note that the slope I of the straight line that describes the relation between $f$ and $V_{\max }$ is homogeneous to a length and is estimated to $\mathrm{I}=10 \mathrm{~mm}$.

\section{Analysis of the experimental results}

\subsection{How an added low-frequency single tone sound reveals the origin of the riser song}

As described in a previous study [21], a low-frequency acoustic field (around $10 \mathrm{~Hz}$ ) leads to a dramatic reduction of the corrugated channel whistling. During the mitigation, as shown below in Figure 18, the very-low frequency carrier modulates the whistling in such a way that it appears only when the remaining low-frequency modulated velocity inside the channel is larger than a certain level.

In the velocity range $20-25 \mathrm{~m} / \mathrm{s}$ measured by the downstream hot-wire, four modes are simultaneously detected by the spectral analysis of the upstream hot-wire signal (see Figure 9). Above $17 \mathrm{~m} / \mathrm{s}$, only one strong mode is highlighted that is to be related to the sudden increase of sound pressure level as shown in Figure 8. This pure tone is checked to be completely extinguishable by a low frequency signal emitted at $14 \mathrm{~Hz}$ by the two loudspeakers as shown on the downstream velocity spectral analysis, and without any obvious modification of the turbulence at the channel exit (Figure 13). As it can be inferred from Figure 13, the injected low frequency acoustic fluctuation level is rather high. In the work reported here, no particular optimization on both frequency and level of the signal imposed to loudspeakers has been performed. 


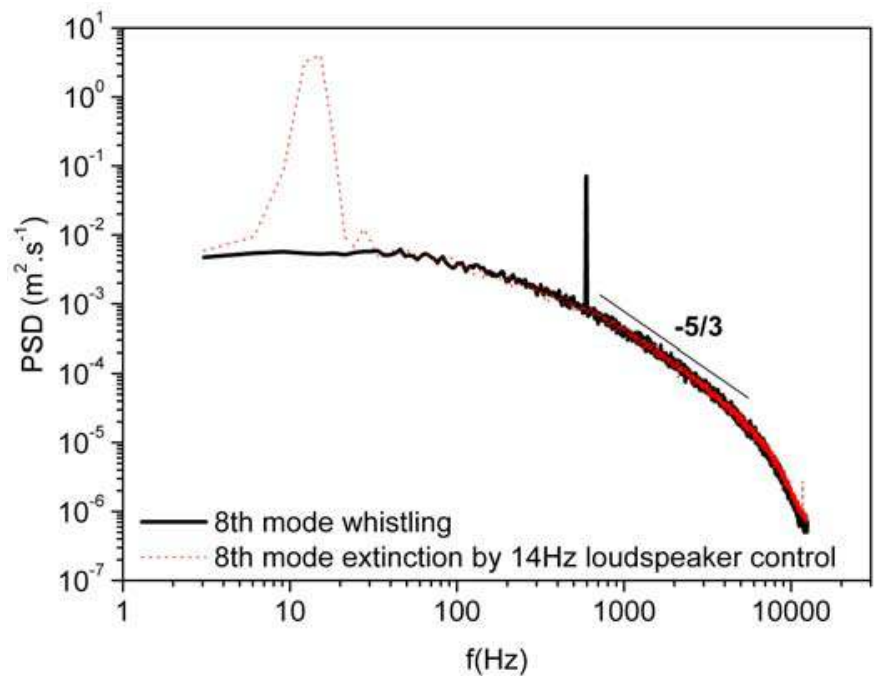

Figure 13. Whistling mitigation (extinction) by a low-frequency acoustic field, downstream hot-wire velocity signal spectral analysis.

Upon analysing this phenomenon, we have observed, as shown below for the velocity signal in Figure 14, very short-time "wave packets" originating at the upstream entry of the channel when the flow speed reaches a sufficient level. These short-time wave packets will be shown to propagate toward the downstream end of the channel at the effective sound celerity, reflect back, propagate upstream and amplify the acoustic field inside the corrugated channel by successive reflections. This observation confirms that a sound source lies close to the pipe's entry section. It may also allow mitigation by controlling the low-frequency acoustic field to limit the amplification through wave superposition of the various reflected wave packets.

Two experimental mitigations at $14 \mathrm{~Hz}$ and $6 \mathrm{~Hz}$ are considered below. In both cases, the rotation speed of the fan is fixed at $37 \mathrm{~Hz}$, allowing an upstream velocity (at $10 \mathrm{~mm}$ in front of the channel entry) of $9.5 \mathrm{~m} / \mathrm{s}$ and inside the channel, near its downstream end, of $19.5 \mathrm{~m} / \mathrm{s}$. For the first one, the frequency of the acoustic mitigation is $14 \mathrm{~Hz}$ and the remaining whistling is significantly reduced. In the second experiment, the mitigation frequency is set to $6 \mathrm{~Hz}$ with a remaining whistling level three times higher than that of the $14 \mathrm{~Hz}$ case. The two signals analysed and compared are the fluid velocity signal delivered by the entry hot wire probe and the acoustic sound pressure signal given by the downstream microphone probe. The results given in Figure 6 show that the acoustic pressure taken at $x_{0}=1951 \mathrm{~mm}$ is close to the maximum value and the minimum sound pressure close to the exit (the pressure acts similarly at the entry) ensures that the velocity fluctuation measured by the hot wire is maximum. In both measurements, the fluctuations created by the waves are then measured with a high signal to noise ratio. The analysis of signal data set is based on the comparison of the fluctuating parts of the flow velocity and acoustic pressure: continuous and low-frequency carrier contributions are removed from both signals. The remaining fluctuations are time frequency analysed and the time-shift correlation between velocity and acoustic pressure is computed.

In both cases $(14 \mathrm{~Hz}$ and $6 \mathrm{~Hz}$ ), the correlation has a maximum for a propagation time of $0.007 \mathrm{~s}$ very close to the time necessary for a wave to propagate from the entry to the exit at $299 \mathrm{~m} / \mathrm{s}$. This is an obvious proof that the noise source is at the entry of the channel. The small fluctuations created at the entry are amplified by successive reflections toward both corrugated channel ends leading to a limited or strong acoustic level depending on the mitigation frequency. 


\subsection{Acoustic mitigation at $14 \mathrm{~Hz}$}

The best mitigation results are obtained for an acoustic frequency close to $14 \mathrm{~Hz}$.

In Figures 14 and 15, as observed in our previous experiment [21], it can be seen that some remaining high frequency fluctuations are superposed over the velocity and pressure carriers. The study of these fluctuations is the core of our analysis.

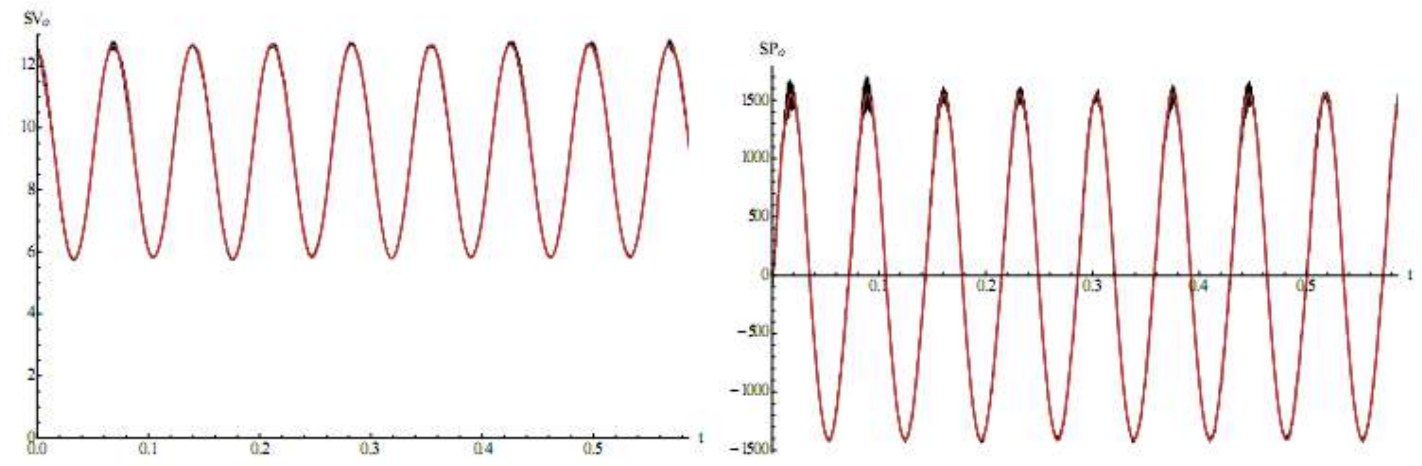

Figure 14. Time records, in $s$, of velocity (left) $S V_{o}(t)$, in $\mathrm{m} / \mathrm{s}$, and sound pressure (right) $S P_{O}(t)$, in $P a$. The black curves are the original data, the red ones represent the identified carrier wave.

The continuous and carrier signals for velocity $V_{c}(t)$ and pressure $P_{c}(t)$ are described as a three term (higher order terms are negligible) Fourier expansion : $\left.\left.V_{c}(t)=A_{0}+A_{1} \cos \left(\omega_{f} t+\phi_{1}\right)\right)+A_{2} \cos \left(2 \omega_{f} t+\phi_{2}\right)\right)$, with $\omega_{f}=2 \pi .14 \mathrm{rad} / \mathrm{s}, A_{0}=9.28 \mathrm{~m} / \mathrm{s}$, $A_{1}=3.46 \mathrm{~m} / \mathrm{s}, \phi_{1}=-6.04 \mathrm{rad}, A_{2}=-0.042 \mathrm{~m} / \mathrm{s}, \phi_{2}=0.13 \mathrm{rad}$ and $P_{c}(t)=B_{0}+B_{1} \cos \left(\left(\omega_{f} t+\varphi_{1}\right)+B_{2} \cos \left(\left(\omega_{f} t+\varphi_{2}\right)\right.\right.$, with $B_{0}=1.4 \quad P a, B_{1}=-1469 P a$, $\varphi_{1}=-4.7 \mathrm{rad}, B_{2}=84.25 \mathrm{~Pa}, \varphi_{2}=-3.51 \mathrm{rad}$. The amplitude and phase coefficients are identified using the standard Mathematica [28] procedure.

It is worth noting that rectified signals $S V_{c}(t)=S V_{o}(t)-V_{c}(t)$ and $S P_{c}(t)=S P_{o}(t)-P_{c}(t)$ in Figure (15) are alike.
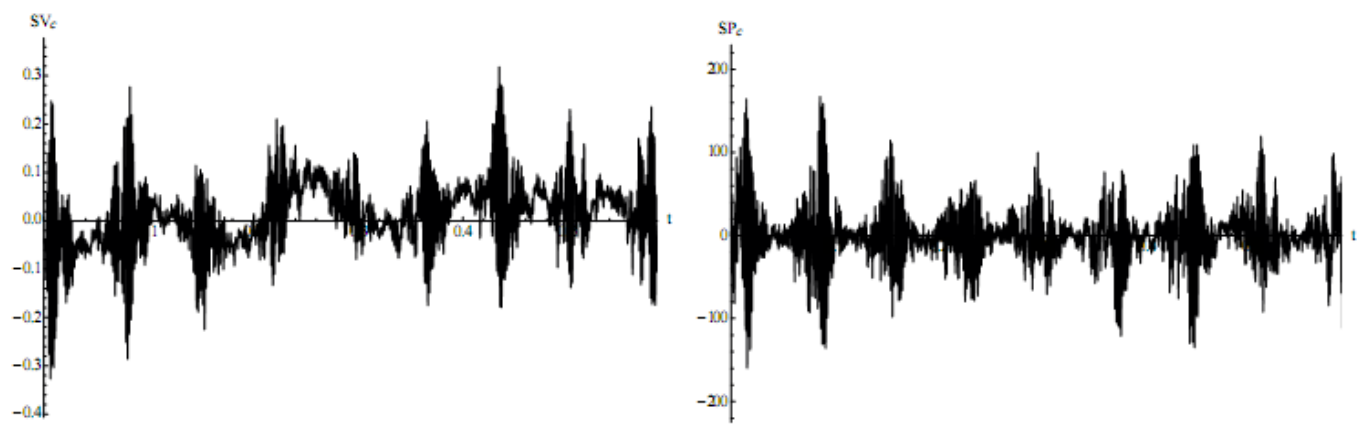

Figure 15. Time records, in $s$, of velocity (left) $S V_{c}(t)$, in $\mathrm{m} / \mathrm{s}$, and sound pressure (right) $S P_{c}(t)$, in $P a$, of the rectified data. 

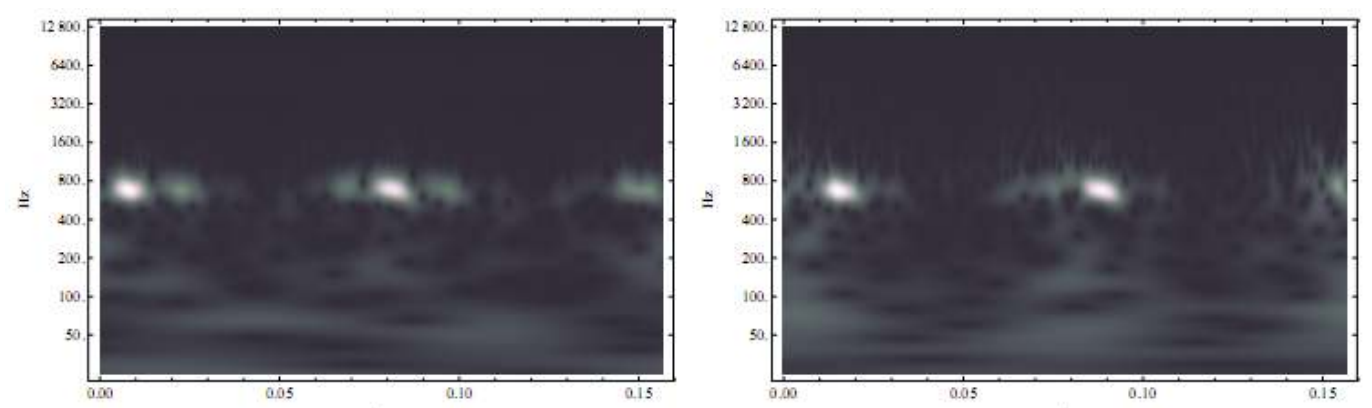

Figure 16. Time-frequency representation of the signal using Gabor wavelet transform of velocity (left) $S V_{c}(t)$ and sound pressure (right) $S P_{c}(t)$.

The time-frequency representation (using a Gabor wavelet of frequency 6 [28]) of the very beginning of both rectified signals is given in Figure 16. This shows, not only an obvious similarity between the two signals, but also a small time shift of the acoustic pressure compared to the velocity. A rough estimate gives this shift to be close to $0.007 \mathrm{~s}$. Since $2 / 299$ $\approx 0.0067 \mathrm{~s}$, one can infer that it is precisely the time necessary for an acoustic perturbation to travel from the entry to the exit. To confirm this, one computes the Pearson correlation coefficient ([28]) between $S P_{c}(t)$ and $S V_{c}(t+\tau)$ as a function of time delay $\tau$. This correlation has been computed using $2^{17}$ samples on both signals. As can be seen in Figure 17, the maximum value of correlation $r$, of about $|r|=0.45$, is reached at time $\tau=0.00689 \mathrm{~s}$. This flying time is precisely that estimated by considering that the acoustic sources are located close to the entry of the channel. It is worth noting that the correlation fluctuates strongly at $669 \mathrm{~Hz}$ that is at the frequency of the remaining whistling.
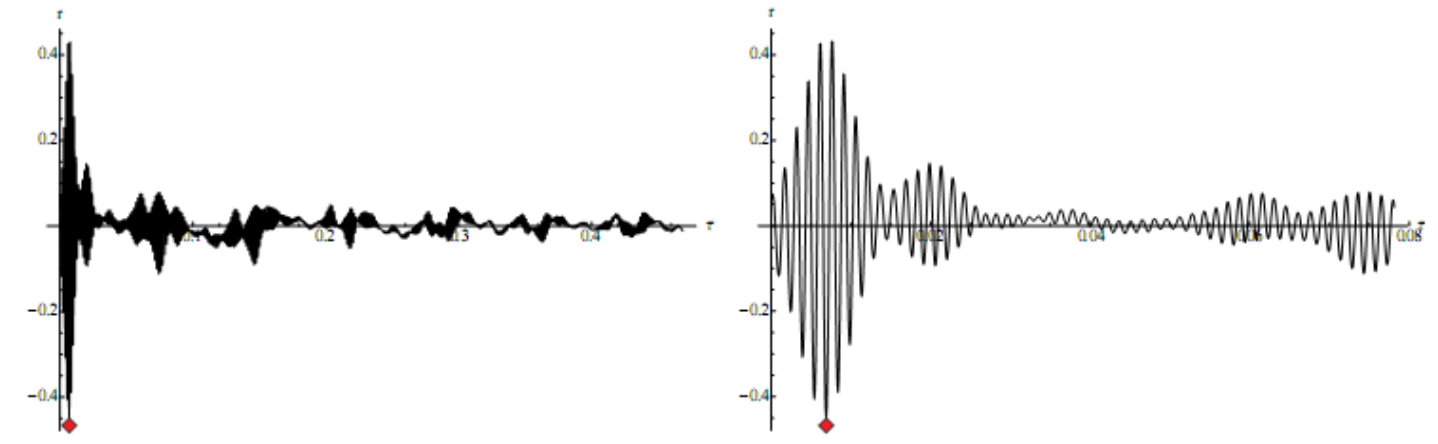

Figure 17. Pearson's correlation coefficient for about half a second delay (left) and 0.1 second delay (right). The diamond marks the maximum absolute value.

\subsection{Acoustic mitigation at $6 \mathrm{~Hz}$}

Here also, the continuous and carrier signals for velocity $V_{c}(t)$ and pressure $P_{c}(t)$ are described as a three term (higher order terms are also negligible) Fourier expansion: $\left.\left.V_{c}(t)=A_{0}+A_{1} \cos \left(\omega_{f} t+\phi_{1}\right)\right)+A_{2} \cos \left(2 \omega_{f} t+\phi_{2}\right)\right)$, with $\quad \omega_{f}=2 \pi .6 \mathrm{rad} / \mathrm{s}$, $A_{0}=9.34 \mathrm{~m} / \mathrm{s}, \quad A_{1}=2.25 \mathrm{~m} / \mathrm{s}, \quad \phi_{1}=2.55 \mathrm{rad}, \quad A_{2}=0.03 \mathrm{~m} / \mathrm{s}, \quad \phi_{2}=-0.22 \mathrm{rad} \quad$ and $P_{c}(t)=B_{0}+B_{1} \cos \left(\omega_{f} t+\varphi_{1}\right)+B_{2} \cos \left(\omega_{f} t+\varphi_{2}\right), \quad$ with $\quad B_{0}=0.87 \mathrm{~Pa}, \quad B_{1}=582.2 \mathrm{~Pa}$, $\varphi_{1}=5.93 \mathrm{rad}, B_{2}=33.56 \mathrm{~Pa}, \varphi_{2}=-5.9 \mathrm{rad}$. The amplitude and phase coefficients are also identified using the standard Mathematica [28] procedure. It is worth noting that again rectified signals $S V_{c}(t)=S V_{o}(t)-V_{c}(t)$ and $S P_{c}(t)=S p_{o}(t)-P_{c}(t)$ in Figure 19 are alike. 

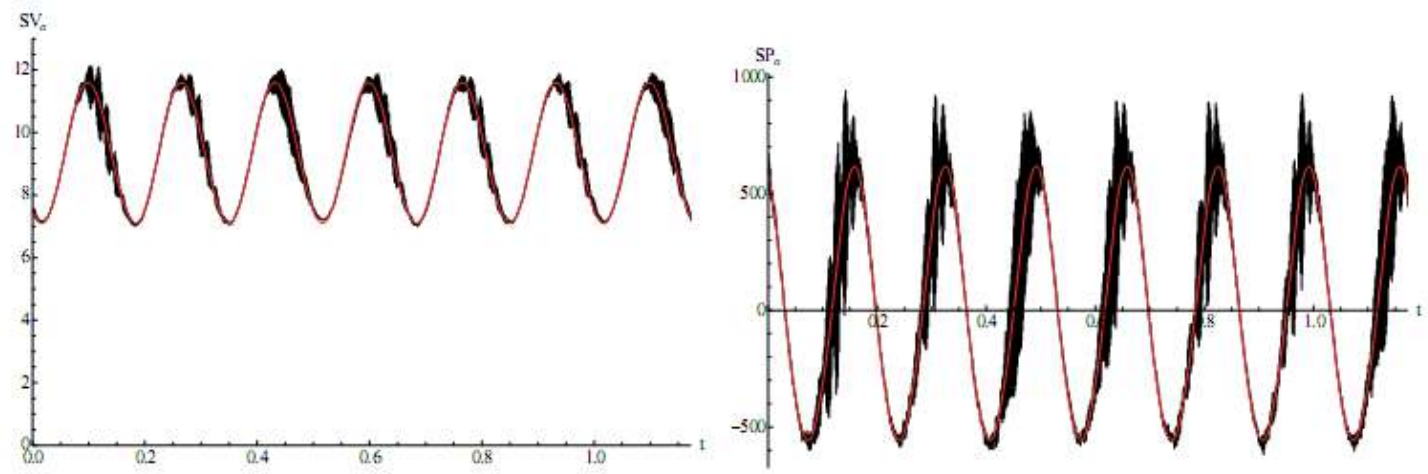

Figure 18. Time records, in s, of velocity (left) $S v_{o}(t)$, in $\mathrm{m} / \mathrm{s}$, and sound pressure (right) $S p_{o}(t)$, in $P a$. The black curves are the original data, the red ones represent the identified carrier wave.

The frequency representation (using again a Gabor wavelet of frequency 6) of the very beginning of both rectified signals is given in Figure 20. This shows, not only a short shift of the acoustic pressure compared to the velocity similar to that observed for the $14 \mathrm{~Hz}$ mitigation, but also an interesting fine time structure of the four wave packets shown here (the same time structure can be observed over the complete record). As previously, each "big wave packet" of velocity and pressure is separated one from the others by again about $0.007 \mathrm{~s}$, again corresponding to the time necessary for a wave to travel from the entry to the exit. Each big packet is made of a set of shorter packets each separated from the previous and following ones by a time of about $0.014 \mathrm{~s}$ (i.e. twice the flying time to the channel exit). Each of it has an intensity that increases until it reaches a maximum and decreases after that maximum. The explanation is that a small wave packet is emitted at the entry by the flow-cavity interaction. It propagates toward the exit end, at an apparent velocity of about $300 \mathrm{~m} / \mathrm{s}$ (taking $0.007 \mathrm{~s}$ to reach the exit). It is reflected and back propagated to the entry. It is reflected again and increased in intensity by coupling with the entry source, and so on, until the entry source vanishes because of the flow mitigation. After that, the wave packet continues to reflect at both ends of the corrugated channel, but loses part of its energy by sound radiation and thus decreases in amplitude. For $14 \mathrm{~Hz}$, this phenomenon is less evident due to the shorter time period of the mitigation.
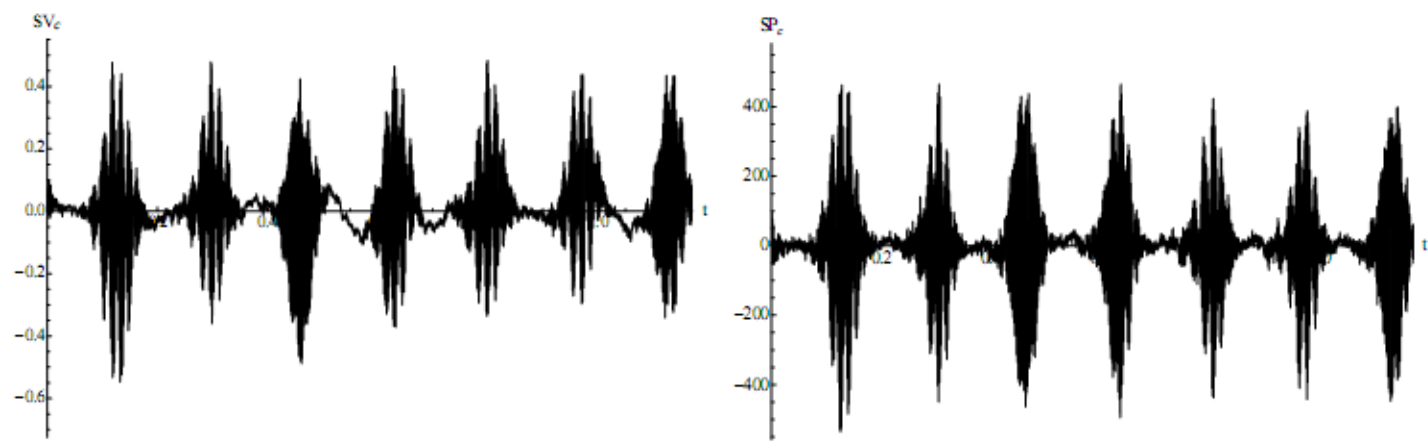

Figure 19. Time records, in $s$, of velocity (left) $S V_{c}(t)$, in $\mathrm{m} / \mathrm{s}$, and sound pressure (right) $S P_{c}(t)$, in $P a$, of the rectified data. 

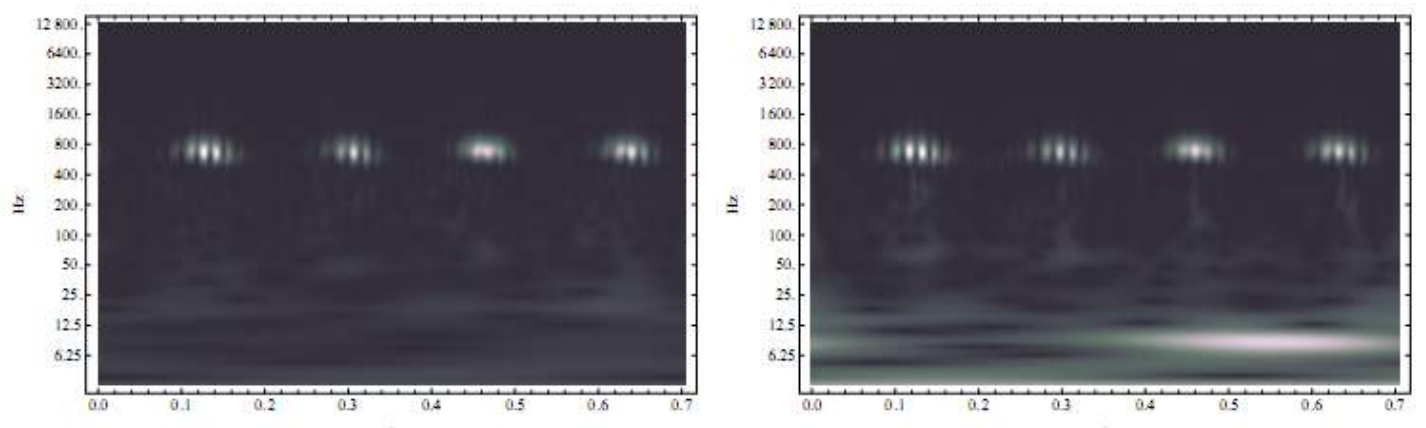

Figure 20. Time-frequency representation of the signal using Gabor wavelet transform of velocity (left) $S V_{c}(t)$ and sound pressure (right) $S P_{c}(t)$.
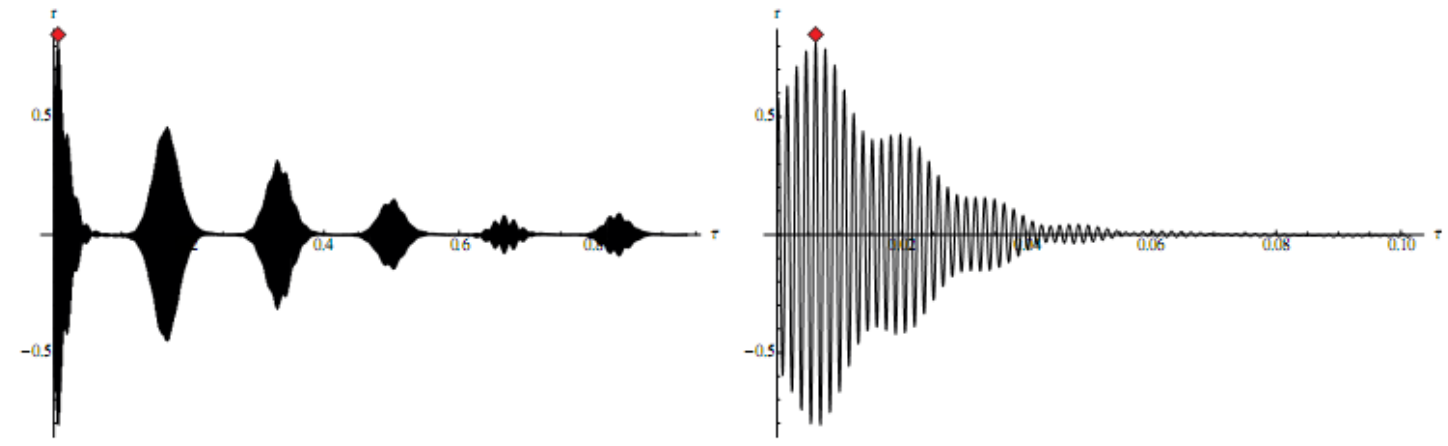

Figure 21. Pearson's correlation coefficient for about one second delay (left) and 0.1 second delay (right). The diamond marks the maximum absolute value.

The Pearson correlation between $S P_{c}(t)$ and $S V_{c}(t+\tau)$ as a function of $\tau$ is again computed using $2^{17}$ samples on both signals. The result is presented in Figure 21. One obtains the maximum value of Pearson's correlation $r$ of about $|r|=0.82$ at time $\tau=0.0062 \mathrm{~s}$. Again, this flying time is very close to that estimated if the acoustic sources are located close to the entry of the channel. The correlation is as twice bigger than previously. This is due to whistling that has much more time to be set on because the acoustic/flow mitigation is almost twice slower than previously and gives time to the acoustic field to take place inside the channel. It is worth noting that the correlation fluctuates strongly again at $669 \mathrm{~Hz}$, that is at the frequency of the remaining whistling.

The acoustic mitigation could be optimized according to that fact. The frequency should be chosen in order to limit the wave reflection at the ends of the channel.

\section{Lattice Boltzmann simulation}

This section presents a numerical study based on the use of the Lattice Boltzmann method (LBM) to predict the noise emission from the corrugated channel flow. The Lattice Boltzmann method differs from traditional patterns by numerically solving an equation based on the physical statistics. Today, although the Lattice Boltzmann method is well established in the domain of Computational Fluid Mechanics, there are few studies based on its acoustic performance. Although it is limited to weakly compressible flows, this method is unsteady and compressible; thereby it can be used to simulate the acoustic waves generated by a turbulent flow.

The present approach is a pragmatic one, as it consists in using the Fourier analysis on the results issuing from a Lattice Boltzmann computation to extract acoustic components from the 
flow data. This Lattice Boltzmann computation is performed by using the Palabos open source code (http://www.palabos.org).

\subsection{Geometry of the computed problem}

The geometry for the Lattice Boltzmann numerical simulation concerns a two-dimensional plane channel of $210 \mathrm{~mm}$ long corrugated on both inner sides with 10 square cavities of dimensions $10 \times 10 \mathrm{~mm}^{2}$ and 11 square corrugations of size $10 \times 10 \mathrm{~mm}^{2}$ (see Figure 22).

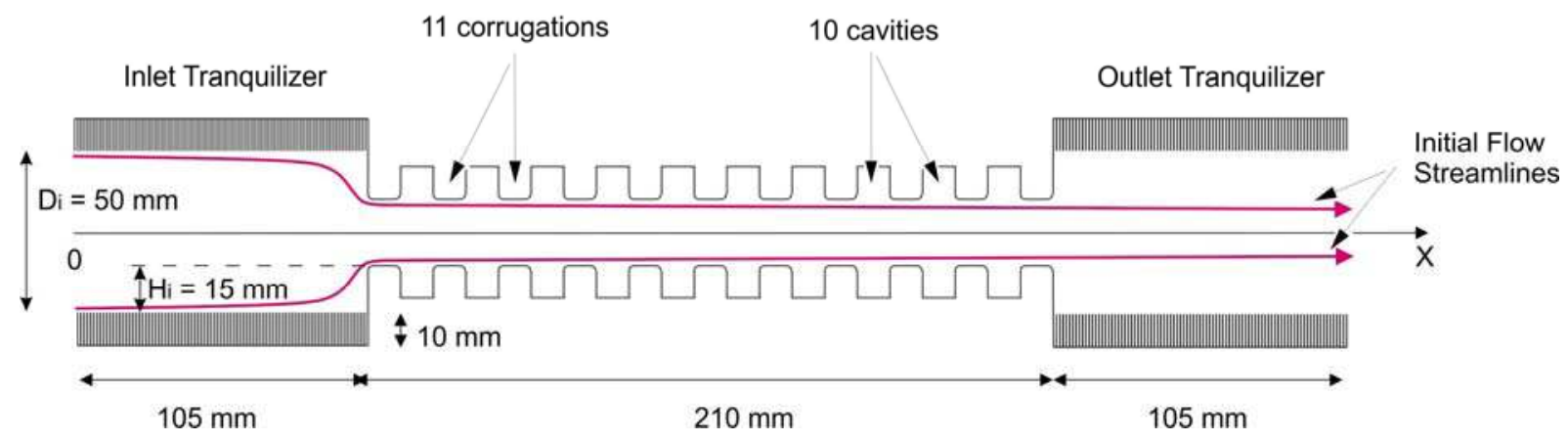

Figure 22. Geometrical arrangement for the Lattice Boltzmann simulation.

The upstream and downstream edges of the corrugations are rounded with a radius equal respectively to $1 \mathrm{~mm}$ and $3 \mathrm{~mm}$. The distance between two facing corrugations is $20 \mathrm{~mm}$. This computed channel is a two-dimensional representation of the experiment detailed in the previous section where the length of the corrugated part is reduced from $2 \mathrm{~m}$ to $210 \mathrm{~mm}$ in order to reduce the computational effort. In the upstream and downstream regions of this corrugated part, there are two tranquilizer chambers of $105 \mathrm{~mm}$ long and $50 \mathrm{~mm}$ high. The role of these tranquilizer chambers consists in introducing a flow step at the inlet and outlet sections of the channel to simulate a channel with acoustically open flanged ends. At the air inlet, this upward step reduces sharply the section of the inflow of $60 \%$, it results in an acceleration of the fluid velocity and a separation of the flow from the walls: A separation bubble forms at the air inlet that reattaches to the wall a few centimeters downstream in the region of the first corrugations. The walls of the tranquilizer region are made of a multitude of very thin $(\approx 0.1 \mathrm{~mm})$ and deep $(10 \mathrm{~mm})$ cavities. These cavities are thin enough so that no flow structure can develop in their interior. Their low thickness promotes viscous effects: their role is to attenuate the acoustic modes in the tranquilizers. The X-axis is classically in the middle of the domain with its origin at the left end side.

\subsection{The Lattice Boltzmann Method}

The Lattice Boltzmann method is implemented on the domain presented in Figure 22. At the entrance of the domain $(x=0)$, there is a non-homogeneous Dirichlet boundary condition for the fluid velocity. The bounce-back approach is applied for this boundary condition. At the outlet $(x=420 \mathrm{~mm})$ a Neumann boundary condition is applied for the fluid velocity. Accurate initial conditions are crucial in the Lattice Boltzmann simulation. Therefore, a typical approach is to use an equilibrium distribution function of the fluid velocity. In the present numerical simulation, the initial flow in the channel is given by the classical $1 / 7^{\text {th }}$ power law turbulent mean velocity profile [29]. In the upstream tranquilizer chamber the flow streamlines are contracted to adapt the flow to the change of section. This contraction is performed by using a shape-function $S_{y}$. The $1 / 7^{\text {th }}$ power law turbulent mean velocity profile 
is multiplied by this shape-function whose role is to "curve" the streamlines to adapt smoothly the flow to the change of section. This shape function acts from the middle of the first tranquilizer chamber $(x \approx 50 \mathrm{~mm})$ where the section height is $D_{i}=50 \mathrm{~mm}$ to the entrance of the channel $(x=105 \mathrm{~mm})$ where the section height is $D=20 \mathrm{~mm}$. This shape function is given for the flow lowest streamline $\left(y=-D_{i} / 2\right.$ in the entrance domain):

$$
S_{-D_{i} / 2}(\xi): \xi \longrightarrow y=\frac{H_{i}}{2}\left(\sin \left(\xi^{4}-\frac{\pi}{2}\right)+1\right)-\frac{D_{i}}{2} ; \text { where }: 0<\xi<\sqrt[4]{\pi}
$$

where $H_{i}=15 \mathrm{~mm}$ is the upward step at the entrance of the channel. A scale factor is applied in order to match $\xi=0$ with roughly the middle of the upstream tranquilizer domain $(x=50 \mathrm{~mm})$, and $\xi=\sqrt[4]{\pi}$ with the entrance of the corrugated part $(x=105 \mathrm{~mm})$. The function $S_{-D_{i} / 2}(\xi)$ performs rising of the lowest streamline, equal to the value of the upward step $H_{i}$ over the distance $50 \mathrm{~mm}$. The highest streamline $S_{+D_{i} / 2}(\xi)$ is the symmetric of $S_{-D_{i} / 2}(\xi)$ with respect to the channel axis. For the streamlines which are between these two extreme streamlines, the shape-function $S_{y}(\xi)$, with $-D_{i} / 2<y<D_{i} / 2$, is calculated by linear interpolation between the functions $S_{-D_{i} / 2}(\xi)$ and $S_{+D_{i} / 2}(\xi)$. The x-component $V_{x}$ of the fluid velocity in the change of section is given by the flow conservation. The y-component of the velocity $V_{y}$ in this change of section is given by the derivation of the shape-function $\partial_{\xi} S_{y}(\xi)$. The shape-function $S_{y}(\xi)$ ensures a smooth initialization for the flow at the change of section and reduces the compressibility errors on this initial flow. This wariness is essential for providing accurate initial conditions.

The collision model for the numerical simulation is the Smagorinsky eddy-viscosity model [30] which assumes that the turbulence small scales induce a viscosity correction proportional to the norm of the strain-rate tensor $v_{t}(x, t)=\left(C_{s} \Delta\right)^{2}\|S(x, t)\|$, where $\|S(x, t)\|=\sqrt{S_{i j} S_{i j}}$ is the magnitude of the strain-rate tensor, $\Delta$ is the bandwidth of the LES filter and $C_{S}$ is a nondimensional coefficient called the Smagorinsky constant: It is an adjusted parameter ensuring the numerical stability of the model by increasing the viscosity as the rate-of-strain of the flow increases. The value of this Smagorinsky coefficient ranges from 0.1 to 0.2 [31]. For our numerical simulation, the value of the Smagorinsky coefficient that seems to be most convenient in terms of flow description is $C_{S}=0.12$.

\subsection{General data for the computed LB model}

The Palabos code works with specific Lattice Boltzmann discrete units [32]. As a first step, the physical system must be converted to a dimensionless one (Table 1 in Appendix 1). As a second step, this dimensionless problem is converted to discrete units. The dimensions of the data are in this last step specific to the Lattice Boltzmann model. A characteristic length $L_{0}$ and a characteristic time delay $T_{0}$ are defined as follows: $L_{0}=P_{t}$ is the length of one corrugation plus one cavity; this is the pitch herein. The characteristic time delay is $T_{0}=P_{t} / V_{0}$, this is the delay for the fluid particles to cross the pitch $P_{t}$. The Mach number of the flow is $M=V_{0} / c_{\text {eff }}$. Considering the sound celerity on the lattice $c_{L B}=1 / \sqrt{3}$ and the 
flow velocity on the lattice $u_{L B}$, the Mach number is expressed in Lattice Boltzmann units as $M=u_{L B} / c_{L B}$. The fluid velocity on the lattice $u_{L B}$ is thus calculated from these two definitions of the Mach number: $u_{L B}=\frac{c_{L B}}{c_{\text {eff }}} V_{0}$.

The flow is computed during $T_{L B}=120$ in the discrete Lattice Boltzmann units. The data of the computed problem are listed in Appendix 1 (Table 1). This computation results in $0.7 \mathrm{~s}$ of flow for five days of computation using one processor (10 cores) of a computer with eight processors Intel Xeon E7-8870 running at $2.4 \mathrm{GHz}$. The sampling rate for the data output is $37300 \mathrm{~Hz}$.

\section{Lattice Boltzmann results and discussion}

The results of this Lattice Boltzmann simulation consist in a fluid density $\rho$ and a fluid velocity vector $\mathrm{V}$ at each point of the mesh grid (Table 1, Appendix 1), at each time step. All these results are in discrete Lattice Boltzmann units. In the present section, three observation domains are defined. $\Omega_{1}$ is a discrete set of 100 measurement points $M i=\left(x_{i}, 0\right)(i=1, \ldots, 100)$ uniformly distributed along the axis of the channel from the entrance $(x=0)$ to the outlet of the domain $(x=420 \mathrm{~mm}) . \Omega_{1}$ represents an antenna of microphones partitioned on the axis, it is used to identify mode-shapes of the acoustic pressure inside the channel. $\Omega_{2}$ is a rectangular domain corresponding to the first cavity located at the inlet of the corrugated part. $\Omega_{3}$ is a domain covering two cavities located in the middle of the corrugated part. $\Omega_{2}$ and $\Omega_{3}$ are two domains on which the flow behavior is investigated and compared to the PIV measurements.

The first comparison between the simulation and the experiment focuses on the interior acoustic field. Let us define an arbitrary reference point $M_{\text {ref }}$ located in the middle of the corrugated part. A Welch analysis is performed on the antenna $\Omega_{1}$. The results of this Welch analysis are the cross-spectrum for the fluid density $S_{\rho}\left(x_{i}, M_{\text {ref }}, f\right)$ and the cross-spectrum for the fluid axial velocity $S_{V x}\left(x_{i}, M_{r e f}, f\right)$ between the points $M_{i}=\left(x_{i}, 0\right)$ and $M_{r e f}$, at frequency $f$. For the analysis of the results, the value of $f$ issuing from this Welch analysis of the Lattice Boltzmann results is expressed in dimensionless units. The dimensioning factor is the first eigen-frequency $f_{0}$ of a corrugated domain of length $L_{T}$ with Neumann-Dirichlet boundary conditions (here $f_{0}=c_{\text {eff }} /\left(4 L_{T}\right) \approx 178 \mathrm{~Hz}$ ). Figure 23 presents the sum of all the 100 spectra of the fluid density $\sum_{i} S_{\rho}\left(x_{i}, M_{r e f}, f\right)(i=1, \ldots, 100)$ calculated on the antenna $\Omega_{1}$ and plotted as a function of the dimensionless frequency $f / f_{0}$. The frequency peaks plotted in Figure 23 correspond roughly to the first eigen-modes of a corrugated domain of length $L_{T}$ with Neumann-Dirichlet boundary conditions. The $n$-th eigen-frequency of this corrugated domain of length $L_{T}$ with Neumann-Dirichlet boundary conditions is equal to $(2 n-1) f_{0}$ [22]. The three frequencies highlighted in Figure 23, corresponding to the modes 1, 4 and 6 , are $1.1 f_{0}, 7 f_{0}$, and $11 f_{0}$ in agreement with the expected ones according to the spectral resolution. This confirms that, for the first eigen-frequencies, the computed channel behaves as a corrugated domain of length $L_{T}$ with Neumann-Dirichlet boundary conditions. 


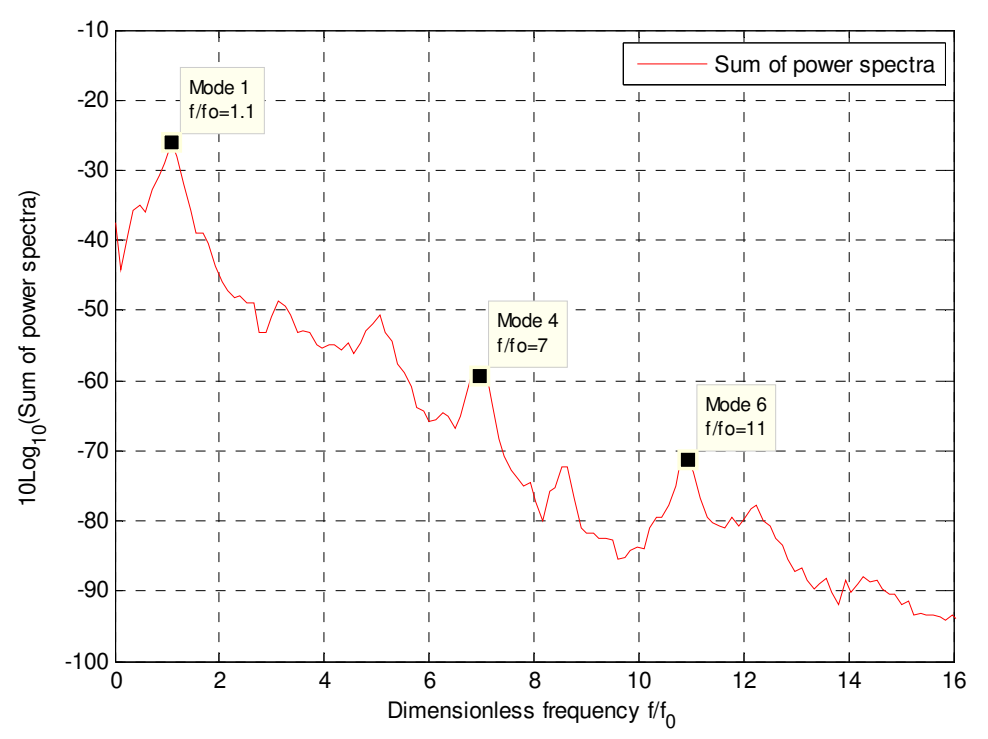

Figure 23. Normalized cross-spectrum of the fluid density $\rho$.

The frequency peaks plotted in Figure 23 do not correspond to the experimental frequency peaks presented in Figure 9. At the velocity used for the computation (ie $V_{0}=16 \mathrm{~ms}^{-1}$ ) Figure 9 presents an experimental maximum sound pressure emission at the sixth mode of the channel $\left(f / f_{0}=6\right)$ meanwhile Figure 23 shows a maximum peak at the first mode of the domain $f / f_{0}=1.1$. There are too many differences in the geometry (length of the domains) and in the boundary conditions between the experiment and numerical simulation to conclude on these mismatches. No matter these mismatches, the numerical example proposed in the present paper must be considered more as a way to analyze the physical phenomena involved in the whistling than as an exact representation of the experiment.

Figures 24, 25 and 26 present the fluid density spectrum $S_{\rho}\left(x_{i}, M_{r e f}, f\right)$ (top figures) and the fluid axial velocity spectrum $S_{V x}\left(x_{i}, M_{r e f}, f\right)$ (bottom figures) plotted as a function of $x_{i}$ at three frequencies: the first frequency peak observed on the system $f=1.1 f_{0}$ (mode 1 ), a medium frequency $f=7 f_{0}$ (mode 4 ) and a high frequency $f=11 f_{0}$ (mode 6 ). The vertical red lines appearing in Figures 24, 25 and 26 delimit the positions of the inlet tranquilizer chamber $(x<105 \mathrm{~mm})$, the corrugated part $(105 \mathrm{~mm} \leq x \leq 315 \mathrm{~mm})$ and the outlet tranquilizer region $(x>315 \mathrm{~mm})$. 

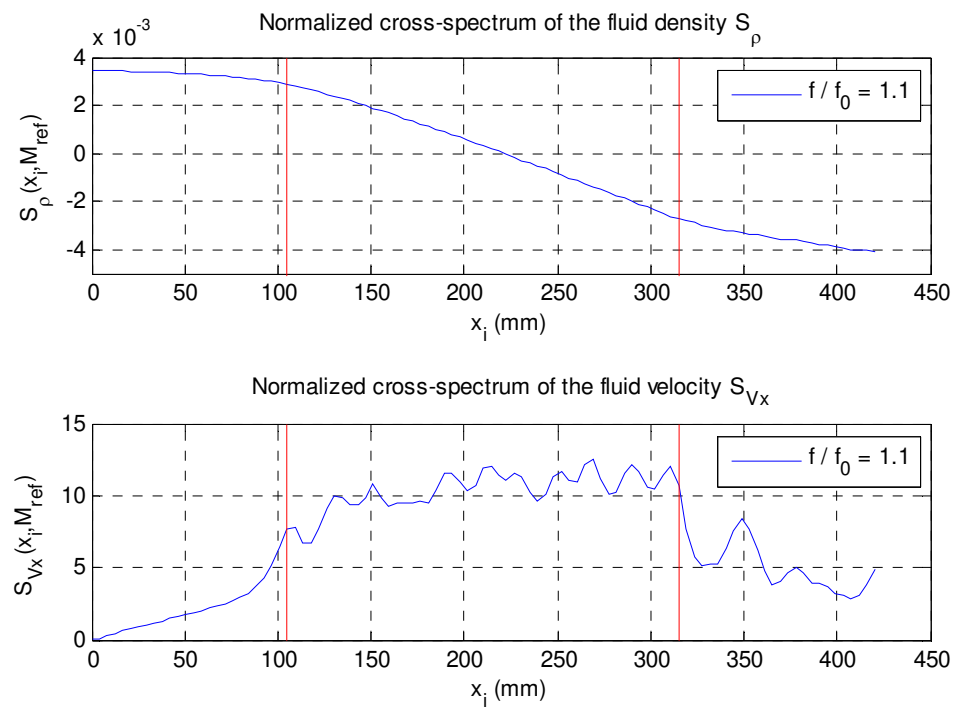

Figure 24. Normalized cross-spectra for the frequency $f / f_{0}=1.1$
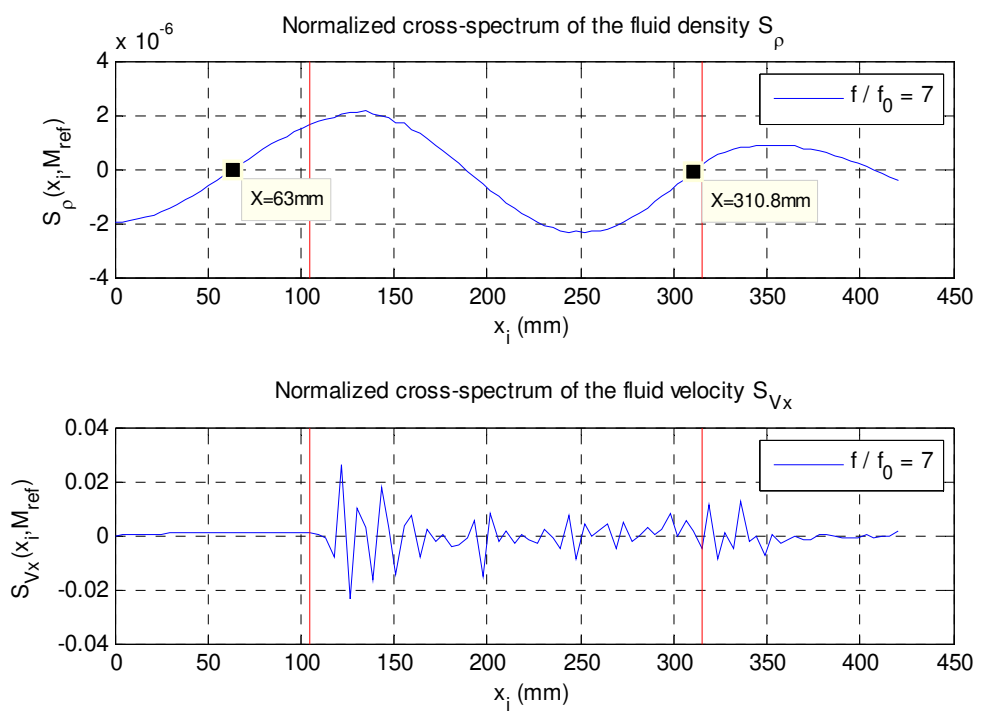

Figure 25. Normalized cross-spectra for the frequency $f / f_{0}=7$ 

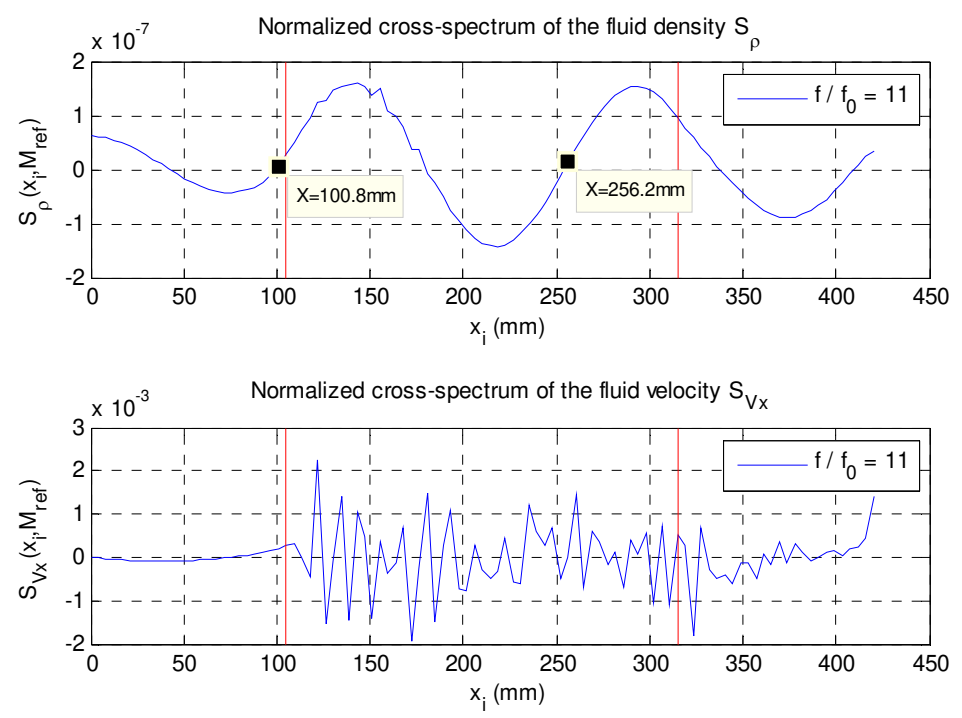

Figure 26. Normalized cross-spectra for the frequency $f / f_{0}=11$

The sound celerity $c$ inside the corrugated part is calculated from the measurement of the wavelengths $\lambda$ that appear in Figures 25 and 26 by using the well-known formula $c=f \lambda$ which gives:

Figure 25

$$
c_{1}=\lambda_{1} f_{1}=1240 \frac{310.8-63}{1000}=307.27 \mathrm{~m} / \mathrm{s},
$$

Figure 26

$$
c_{2}=\lambda_{2} f_{2}=1946 \frac{256.2-100.8}{1000}=302.41 \mathrm{~m} / \mathrm{s} \text {. }
$$

The sound celerity calculated from the wavelength measurements in Figures 25 and 26 is very close to the experimental value $c_{\text {eff }}=299 \mathrm{~m} / \mathrm{s}$. This result confirms that the mode-shapes observed on the density power spectrum in Figures 25 and 26 are indeed acoustic modes.

One of the major problems encountered in this numerical simulation comes from the fact that there is no acoustic absorbing boundary condition proposed in the present Palabos version. So, overcoming the boundary conditions of the corrugated part's outer domains is impossible. Moreover, these boundary conditions are described in terms of fluid mechanics and not for an acoustic behavior. As a result, the eigen-modes observed in Figures 24, 25 and 26 are not eigen-modes of a channel with open ends, but eigen-modes of a channel with unknown acoustic boundary conditions. Nevertheless, these acoustic boundary conditions can be estimated visually from the shape of the function $S_{\rho}$ presented in Figures 24, 25 and 26. These functions $S_{\rho}$ correspond to a Neumann acoustic boundary condition at the inlet of the domain $(x=0)$ : the non-homogeneous bounce-back method that has been used at the inlet to create the incoming flow seems to induce at this section an acoustic Neumann boundary condition. Note that the bounce-back method is also used to model the walls of the corrugated part for which a Neumann boundary condition is also expected. The outflow condition is a zerogradient boundary condition for the fluid velocity vector $V$. The acoustic boundary condition induced by this outflow boundary condition cannot be identified clearly from the observation of the mode-shapes in Figures 24, 25 and 26. The velocity spectra $S_{V x}$ plotted on the bottom of Figures 24,25 and 26 are very noisy. It is likely that the large band turbulence contribution to the spectrum $S_{V x}$ masks the much lower acoustic one. This behavior also appears on the 
experiment: in Figure 8, the downstream hot-wire measurements (white triangles) cannot detect the whistling phenomenon because it is covered by the turbulent noise. As expected, the cross-spectra $S_{\rho}$ and $S_{V x}$ are significantly reduced in the regions of the tranquilization chambers (Figures 24, 25 and 26): the thin and deep cavities which cover the walls of these regions seem to actually play their role of dampers.

The next observations concern images of the vorticity field calculated in the domains $\Omega_{2}$ (first cavity) and $\Omega_{3}$ (middle of the corrugated part). In $\Omega_{2}$ domain, Figures 27a and b illustrate the vena contracta phenomenon that occurs at the inlet of the corrugated part where the flow separates from the wall. At this flow separation, vortex structures are created in the region very close to the inlet. These structures grow along the separation bubble and detach at a somewhat regular frequency in the region close to the upstream rounded cavity edge before being carried downstream by the flow. Another noticeable phenomenon is the two contrarotating vortices inside the cavity, and the expulsion of fluid from the upstream rounded cavity edge that feeds the region of the separation bubble.

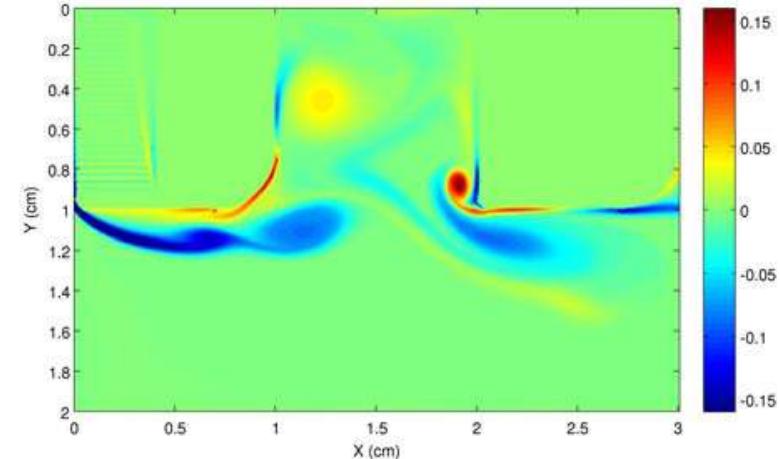

(a)

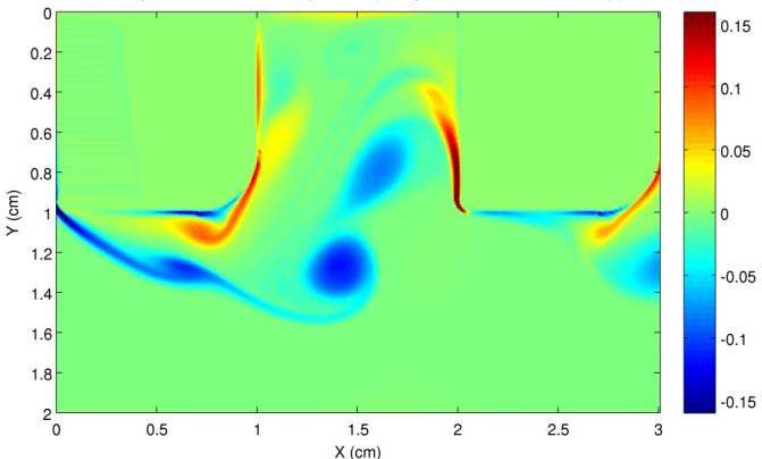

(b)

Figure 27. Vorticity in the inlet section (domain $\Omega_{2}$ ). (a) at time $0.43124 \mathrm{~s}$, (b) at time $0.43237 \mathrm{~s}$, (see associated video vorticity_inlet.avi)

Figures 28 (a-b-c-d) present the vorticity field computed in the middle section of the corrugated part for equal time steps. Comparing Figures 27 and 28 and associated videos, it can be noticed that, in the middle section of the corrugated part, the vorticity is lower than in the inlet region, and that the vortices inside the cavity are more regular. In Figure 28a, a vortex structure that just enters the domain $\Omega_{3}$ at time $t_{1}=0.42785 \mathrm{~s}$ is observed, it crosses the domain $\Omega_{3}$ in Figures $28 \mathrm{~b}$ and $28 \mathrm{c}$ and leaves it at time $t_{2}=0.43124 \mathrm{~s}$, in Figure $28 \mathrm{~d}$. The domain $\Omega_{3}$ is $40 \mathrm{~mm}$ long, so the convection velocity $U_{c}$ of this vortex can be calculated: $U_{c}=0.04 /(0.43124-0.42785)=11.8 \mathrm{~m} / \mathrm{s}$. Similarly, the convection velocity at the cavity mouth can be estimated experimentally from velocity $V_{\max }$ in the cavity (\$4) and mean velocity $U$ on the corrugated part axis $(\$ 2)$. In the region of cavity mouth, the velocity is identified as the inside vortex maximal velocity $V_{\max }$, so that in the shear layer $U_{c}=\left(V_{\max }+U\right) / 2$. The results are presented in Figure 29. It is shown that the convection velocity deduced from the LBM simulation is quite in a good agreement with its experimental estimation for $U=16 \mathrm{~m} / \mathrm{s}$. Moreover, the convection velocity is linearly related to the velocity on the axis. According to numerical results, vortices are regularly emitted from the rounded edge and cross the cavity mouth over a distance equal to the pitch length $P_{t}$. Consequently an emission frequency can be estimated by $U_{c} / P_{t}$ and it is experimentally obtained to be equal to the whistling frequency $f$ (see Figure 29). 


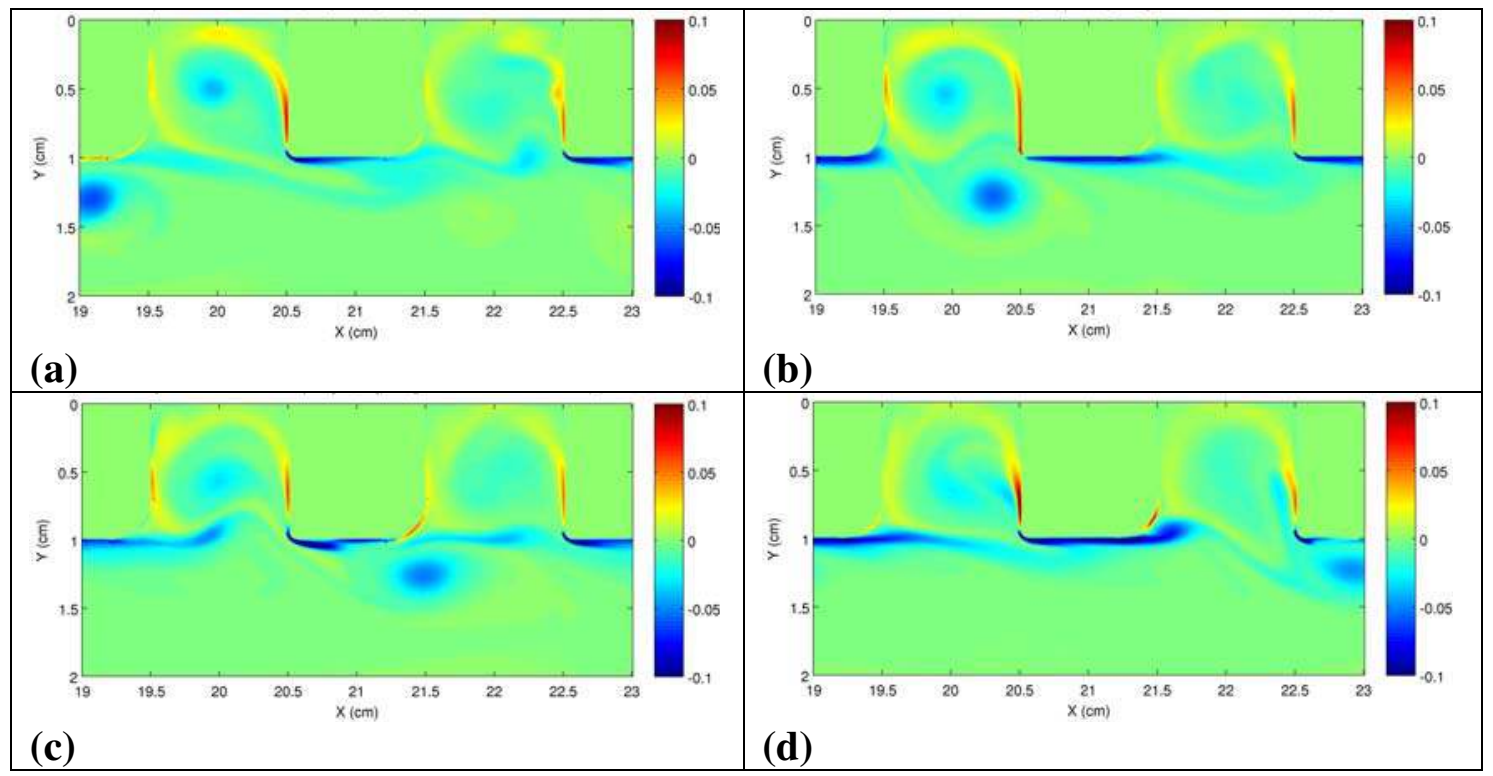

Figure 28. Vorticity in domain $\Omega_{3}$. (a) at time $0.42785 \mathrm{~s}$, (b) at time $0.42898 \mathrm{~s}$, (c) at time $0.43011 \mathrm{~s}$, (d) at time $0.43124 \mathrm{~s}$. (see associated video vorticity_middle.avi)

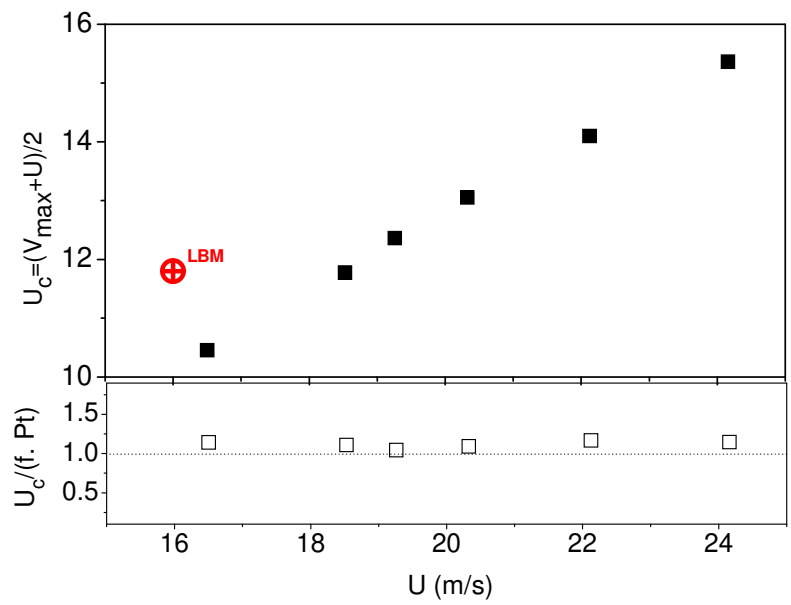

Figure 29. Estimation of convective velocity $U_{c}$ at the cavity mouth and agreement between the upstream edge vortex emission frequency $U_{c} / P_{t}$ and experimental whistling frequency $f$, using PIV and hot-wire velocity measurements.

\section{Final discussion and conclusion}

The present experimental facility and the associated numerical tools were shown to be well suited for investigating flow acoustic situations. A series of combined acoustic sound pressure and fluid velocity measurements were conducted under different configurations of mitigation by a very low frequency acoustic wave. The aim of these experiments was to identify clearly the acoustic sound field that takes place inside the corrugated channel under the flow excitation. A low frequency acoustic mitigation induces a flow speed modulation around the mean velocity. This modulation dramatically changes the nature of the sound fields created by the corrugated channel, from intense tone to much quieter periodic whistling. Under this mitigation, a residual fluctuation was measured on both the acoustic field inside the channel and the flow velocity at its entry. By eliminating the low frequency forcing term on both signals and by correlating them, it was shown that the sound source is not only located near 
the entry end of the corrugated channel but also that the residual acoustic field is due to the superposition of waves reflected at both ends of the channel.

The strong coupling that exists between the acoustics and the flow was also investigated by numerical simulation using the Lattice Boltzmann Method. The results of this numerical simulation were analyzed by a Welch method which permits to identify acoustic eigen-modes of the channel. Limitations arise when analyzing the acoustic behavior because the boundary conditions involved in the lattice Boltzmann schemes are mainly designed for the description of the flow, their acoustic properties are not considered. As a result, the tranquilizer zones located at the inlet and at the outlet of the corrugated part participate strongly to the acoustic eigen-modes of the computed domain. In the present numerical simulation, a solution consisting in a multitude of thin and deep cavities was proposed to create two soundabsorbing regions at the ends of the domain. This method appeared to be of limited efficiency because the mode-shapes present a Neumann boundary condition at the inlet of the domain in place of the expected Dirichlet boundary condition at the inlet of the corrugated part. Recently, some definitions of absorbing layers for nonreflecting acoustic boundary conditions appeared in the literature [33]. An outflow sponge layer is also proposed in the version 1.4 of Palabos, but only for three-dimensional problems, and the present model is a two-dimensional one. The incorporation of such a non-reflective sponge layer in future work should improve greatly the accuracy of the simulation, thus allowing quantitative acoustic comparison with experimental data.

\section{Acknowledgments.}

The work presented herein was supported by Institut Carnot STAR through the CREATUCE Project. The contribution of the mechanics staff of IRPHE in designing and machining the corrugated channel is acknowledged. 


\section{References}

[1] S. Serafin and J. Kojs, "The voice of the dragon: A physical model of a rotating corrugated tube", in Proceedings of the Sixth International Conference on Digital Audio Effects, DAFex-03, London, 8-11 September 2003.

[2] G. Nakiboglu, O. Rudenko and A. Hirschberg. "Aeroacoustics of swinging corrugated tube: Voice of the Dragon”, J. Acoust. Soc. Am., 131, 1, pp. 749-765, 2012.

[3] W. Burstyn, "Eine neue pfeife (a new pipe)", Z. Tech. Phys. Leipzig, 3, pp. 179-180, 1922.

[4] P. Cermak, "Über die tonbildung bei metallschläuchen mit eingedrücktem spiralgang (on the sound generation in flexible metal hoses with spiraling grooves)", Phys. Z., 23, pp. 394$397,1922$.

[5] Lecture Notes on the Mathematics of Acoustics, Chapter 11, edited by M. C. M. Wright, Imperial College Press, London, 2005.

[6] G. Nakiboglu, H.B.M. Manders and A. Hirschberg, "Aeroacoustic power generated by a compact axisymmetric cavity: Prediction of self-sustained oscillation and influence of the depth", J. Fluid. Mech., 703, pp.163-191, 2012.

[7] A. M. Petrie and I. D. Huntley, "The acoustic output produced by a steady airflow through a corrugated duct," J. Sound Vib. 70, pp. 1-9, 1980.

[8] F. S. Crawford, "Singing corrugated pipes," Am. J. Phys. 42, 278-288 1974.

[9] L. H. Cadwell, "Singing corrugated pipes revisited," Am. J. Phys. 62, pp. 224-227, 1994.

[10] U. R. Kristiansen and G. A. Wiik, "Experiments on sound generation in corrugated pipes with flow,” J. Acoust. Soc. Am., 121, pp. 1337-1444, 2007.

[11] J. Golliard, D. Tonon and S. P. C. Belfroid, "Experimental investigation of the source locations in whistling short corrugated pipes," Proc. of the ASME $20103^{\text {rd }}$ joint US-European Fluid Eng. summer meeting. Montreal Canada. Paper FEDSM-ICNMM2010-30732, 2-4 August 2010.

[12] S. P. C Belfroid, R. J. Swindell and N. Kitney, "Flow induced pulsations due to flexible risers," in Offshore Technology Conference, Houston Texas, Report No. OTC 19904, 4-7 May 2009.

[13] V. Debut, J. Antunes and M. Moreira, "Flow-acoustic interaction in corrugated pipes: Time domain simulation of experimental phenomena", in Ninth International Conference on Flow-Induced Vibration, Prague, Czech Republic, pp. 417-425, 2008.

[14] H. Goyder, "On the modelling of noise generation in corrugated pipes", J. Pressure Vessel Technol., 132(4), 041304, 2010.

[15] M. Popescu, S T. Johannesen and S. Wei, "Flow-induced acoustics in corrugated pipes", Commun. Comput. Phys., 10(1), pp. 120-139, 2011.

[16] V. F. Kopiev, M. A. Mironov and V. S. Solntseva, "Sound generation, amplification and absorption by air flow through waveguide with periodically corrugated boundary", in Proceedings of Forum Acusticum, Budapest, Hungary, 29 August - 2 September 2005.

[17] M. S. Howe, Acoustics of fluid-structure interactions, Cambridge Monographs on Mechanics, Cambridge University Press 1998.

[18] D. Mazzoni and U.R. Kristiansen, Aero-acoustics of a flow pipe having a single small cavity. Proceedings of the Forum Acusticum 2011, Aalborg, Denmark, June 27 - July 1, 2011.

[19] U. Kristiansen, D. Mazzoni and A. B. Krogvig, "Aeroacoustic investigation of a flow pipe with a small cavity using the lattice Boltzmann method". Proceedings of the $35^{\text {th }}$ Scandinavian Symposium on Physical Acoustics, Geilo, Norway, January 29 - February 1, 
2012.

[20] B. Rajavel and M. G. Prasad, "Acoustics of corrugated pipes: A review", Appl. Mech. Rev., 65, pp. 050000/1-050000/24, 2013.

[21] U.R. Kristiansen, P-O. Mattei, C. Pinhede and M. Amielh, "Experimental study of the influence of low frequency flow modulation on the whistling behavior of a corrugated pipe", J. Acoust. Soc. Am., 130, pp. 1851-1855, 2011.

[22] A. D. Pierce, Acoustics. An Introduction to its Physical Principle and Applications. McGraw-Hill Book Company, 1981.

[23] J. W. Strutt, Lord Rayleigh, The Theory of Sound. Dover Publications, 1945.

[24] S. Boij, "Flow effects on the acoustic end correction of a sudden in-duct area expansion". J. Acoust. Soc. Am., 126(3), pp. 995-1004, 2009.

[25] W. Duan and R. Kirby, "A hybrid finite element approach to modeling sound radiation from circular and rectangular ducts". J. Acoust. Soc. Am., 131(5), pp. 3638-3649, 2012.

[26] J.W. Elliott, "Corrugated pipe flows". Lecture Notes on the Mathematics of Acoustics. M.C.M. Wright, Ed., Imperial College Press, pp. 207-222, 2004.

[27] G. Nakiboglu, S.P.C. Belfroid, J.F.H. Willems and A. Hirchberg, "Whistling behavior of periodic systems: corrugated pipes and multiple side branch system”. Int. J. Mech. Sci., 52, pp. 1458-1470, 2010.

[28] S. Wolfram, Wolfram Mathematica 9. Wolfram Research Inc., 2012.

[29] L. J. De Chant, "The venerable $1 / 7^{\text {th }}$ power law turbulent velocity profile: a classical non-linear boundary value problem solution and its relationship to stochastic processes". Applied Mathematics and Computation, 161, pp. 463-474, 2005.

[30] J. Smagorinsky, "General circulation experiments with the primitive equations: I. The basic experiment”. Mon. Weather Rev., 91, pp. 99-165, 1963.

[31] J. Meyers and P. Sagaut, "On the model coefficients for the standard and the variational multi-scale Smagorinsky model”. J. Fluid Mech., 569, pp. 287-319, 2006.

[32] J. Latt, "Choice of units in lattice Boltzmann simulations". Technical report, LBMethod.org, 2008.

[33] H. Xu and P. Sagaut, "Analysis of the absorbing layers for the weakly-compressible lattice Boltzmann methods”. J. Comput. Physics, 245, pp. 14-42, 2013. 


\section{Appendix 1}

\begin{tabular}{|l|l|}
\hline In physical units: & \\
\hline Total length of the computed domain & $L_{T}=420 \mathrm{~mm}$ \\
\hline Length of the channel $\mathrm{L}_{p}$ & $L_{p}=210 \mathrm{~mm}$ \\
\hline Distance between two facing corrugations & $D=20 \mathrm{~mm}$ \\
\hline Length of the cavities & $w=10 \mathrm{~mm}$ \\
\hline Depth of the cavities & $H=10 \mathrm{~mm}$ \\
\hline Sound celerity & $c_{\text {eff }}=299 \mathrm{~m} / \mathrm{s}$ \\
\hline Density of the fluid & $\rho=1.2 \mathrm{~kg} / \mathrm{m}^{3}$ \\
\hline Fluid velocity at the center of the channel & $V_{0}=16 \mathrm{~m} / \mathrm{s}$ \\
\hline Kinematic viscosity of the fluid (air) & $v=15.10^{-6} \mathrm{~m}^{2} / \mathrm{s}$ \\
\hline Characteristic length (= pitch) & $P t=20 \mathrm{~mm}$ \\
\hline Mesh grid & $6000 x 1000$ \\
\hline
\end{tabular}

\begin{tabular}{|l|l|}
\hline In dimensionless units: & \\
\hline Reynolds number based upon the pitch length $P t$ & $R e=V_{0} P t / v=13293$ \\
\hline Reynolds number used for the Palabos simulation & $R e=13000$ \\
\hline Time step for the Palabos output & $d t=0.01$ (dimensionless) \\
\hline
\end{tabular}

\begin{tabular}{|l|l|}
\hline In the discrete Lattice Boltzmann units: & \\
\hline Theoretical fluid velocity at the center of the channel & $u_{L B}=0.03$ in LB units \\
\hline Sound celerity & $c_{L B}=1 / \sqrt{3}$ \\
\hline Density of the fluid & $\rho_{L B}=1$ \\
\hline
\end{tabular}

Table 1. Data of the computed problem. 